\title{
Self-intersection numbers of curves on the punctured torus
}

\author{
Moira Chas and Anthony Phillips
}

November 2, 2018

\begin{abstract}
The minimum number of self-intersection points for members of a free homotopy class of curves on the punctured torus is bounded above in terms of the number $L$ of letters required for a minimal description of the class in terms of the generators of the fundamental group and their inverses: it is less than or equal to $(L-2)^{2} / 4$ if $\mathrm{L}$ is even, and $(L-1)(L-3) / 4$ if $\mathrm{L}$ is odd. The classes attaining this bound are explicitly described in terms of the generators; there are $(L-2)^{2}+4$ of them if $L$ is even, and $2(L-1)(L-3)+8$ if $L$ is odd; similar descriptions and totals are given for classes with self-intersection number equal to one less than the maximum.

Proofs use both combinatorial calculations and topological operations on representative curves. Computer-generated data are tabulated counting, for each non-negative integer, how many length- $L$ classes have that self-intersection number, for each length $L$ less than or equal to 12. Experimental data are also presented for the pair-of-pants surface.
\end{abstract}

\section{Introduction}

The punctured torus has the homotopy type of a figure-eight. Its fundamental group is free on two generators: once these are chosen, say $a, b$, a free homotopy class of curves on the surface can be uniquely represented as a reduced cyclic word in the symbols $a, b, A, B$ (where $A$ stands for $a^{-1}$ and $B$ for $b^{-1}$ ). A cyclic word $w$ is an equivalence class of words related by a cyclic permutation of their letters; we will write $w=\left\langle r_{1} r_{2} \ldots r_{n}\right\rangle$ where the $r_{i}$ are the letters of the word, and $\left\langle r_{1} r_{2} \ldots r_{n}\right\rangle=\left\langle r_{2} \ldots r_{n} r_{1}\right\rangle$, etc. Reduced means that the cyclic word contains no juxtapositions of $a$ with $A$, or $b$ with $B$. Note here that we will call a free homotopy class (a reduced cyclic word) primitive if is not a proper power of another class (another word); and among the nonprimitive classes are words we will call pure powers: those which are a proper power of

Key words and phrases: punctured torus, free homotopy classes of curves, self-intersection. 2000 Mathematics Subject Classification: Primary 57M05, Secondary 57N50,30F99. 
a generator. The length (with respect to the generating set $(a, b)$ ) of a free homotopy class of curves is the number of letters occurring in the corresponding reduced cyclic word.

This work studies the relation between length and the self-intersection number of a free homotopy class of curves: the smallest number of self-intersections among all general-position curves in the class. (General position in this context means as usual that there are no tangencies or multiple intersections). The self-intersection number is a property of the free homotopy class and hence of the corresponding reduced cyclic word $w$; we denote it by $\operatorname{SI}(w)$.

Theorem 1.1. The maximal self-intersection number for a primitive reduced cyclic word of length $L$ on the punctured torus is:

$$
\begin{cases}(L-2)^{2} / 4 & \text { if } L \text { is even } \\ (L-1)(L-3) / 4 & \text { if } L \text { is odd }\end{cases}
$$

The words realizing the maximal self-intersection number are (see Figure 1):

(1) L even:

(i) $\left\langle r^{L / 2} s^{L / 2}\right\rangle, r \in\{a, A\}, s \in\{b, B\}$

(ii) $\left\langle r^{i} s^{j} r^{L / 2-i} S^{L / 2-j}\right\rangle, r \in\{a, A\}, s \in\{b, B\}, S=s^{-1}$, and similar configurations interchanging $r$ and $s$.

(2) L odd:

(i) $\left\langle r^{(L+1) / 2} s^{(L-1) / 2}\right\rangle, r \in\{a, A\}, s \in\{b, B\}$, or vice-versa

(ii) $\left\langle r^{i} s^{j} r^{(L+1) / 2-i} S^{(L-1) / 2-j}\right\rangle,\left\langle r^{i} s^{j} r^{(L-1) / 2-i} S^{(L+1) / 2-j}\right\rangle, r \in\{a, A\}, s \in\{b, B\}, S=$ $s^{-1}$, and similar configurations interchanging $r$ and $s$.

Elementary counting with Theorem 1.1 yields the next result:

Theorem 1.2. The number of distinct primitive free homotopy classes of length $L$ realizing the maximal self-intersection number is

$$
\begin{cases}(L-2)^{2}+4 & \text { if } L \text { is even } \\ 2(L-1)(L-3)+8 & \text { if } L \text { is odd }\end{cases}
$$

Elementary computation with Theorem 1.2 allows the inequality to be reversed:

Theorem 1.3. Let $w$ be the reduced cyclic word corresponding to a primitive free homotopy class of curves on the punctured torus. Then if $\mathrm{SI}(w) \geq 1$, the length of $w$ is greater than or equal to the smallest integer larger than $2 \sqrt{\mathrm{SI}(w)}+2$. Moreover, this bound is sharp. 

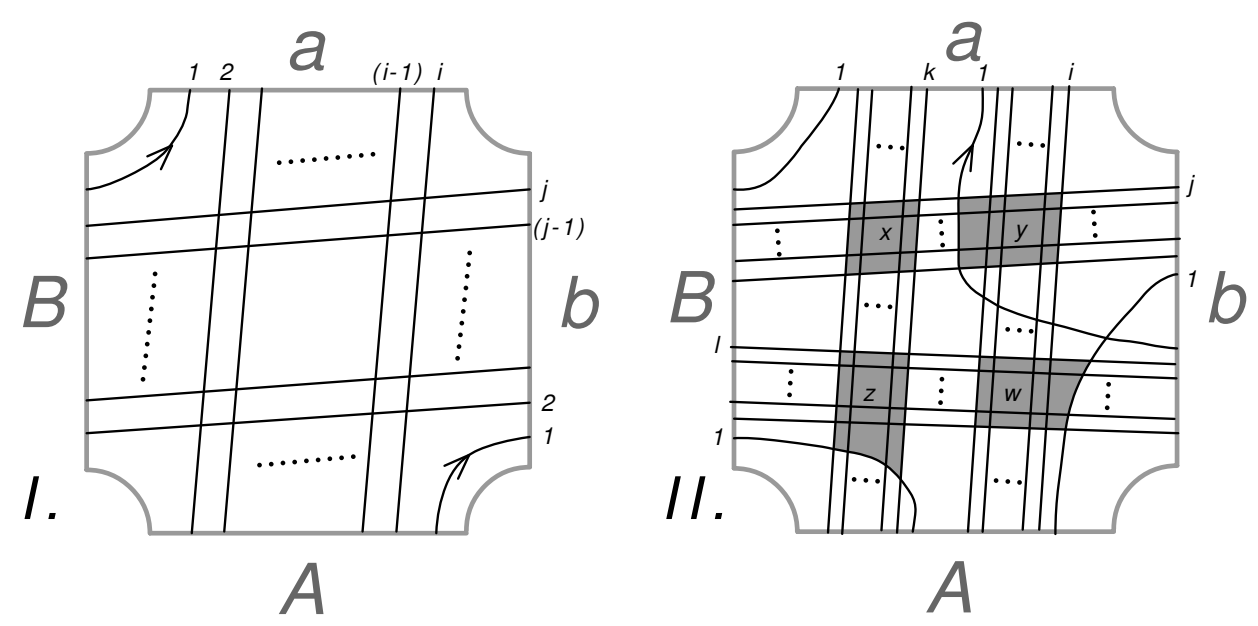

Figure 1: Curves of maximal self-intersection on the punctured torus. I. $w=\left\langle a^{i} b^{j}\right\rangle$ with $(i-1)(j-1)$ intersection points, a maximum when $i=j$ (even length) or $i=j \pm 1$ (odd length). II. $w=\left\langle a^{i} b^{j} a^{k} B^{l}\right\rangle$. Block " $\mathrm{x}$ " has $(k-1)(j-1)$ intersection points; block "y" has $i(j-1)$; block "z" has $l(k-1)$; block "w" has $i(l-1)$; and there are an additional $i$. The total is $(i+k-1)(j+l-1)$, a maximum when $i+k=j+l$ (even length) or $i+k=j+l \pm 1$ (odd length). Graphic conventions from Section 2.1; curve II drawn using the algorithm of [3]. Similar diagrams appear in [7].

Remark 1.4. Pure-power words of length $L$ between 2 and 6 do not fit the pattern of Theorems 1.1 - 1.3. Namely, $\mathrm{SI}\left(r^{L}\right)=L-1>(L-2)^{2} / 4$ and $(L-1)(L-3) / 4$ for integers in that range. Theorems 1.1-1.3 can in fact be extended to all words of length seven or more, primitive or not.

Remark 1.5. The length of the word representing a free homotopy class depends on the choice of generating set $(a, b)$ for the fundamental group, while its self-intersection number does not. Since the theorems above apply for any generating set, they can be rephrased in terms of the shortest of such lengths.

Remark 1.6. The group of automorophisms of the fundamental group of the punctured torus acts on the set of cyclic words with a fixed self-intersection number $n$. Words with maximal self-intersection number minimize length in an orbit of this action. Rivin asked us if every orbit contains a word with maximal self-intersection number for its length. But $w=\langle a b a b A B\rangle$ is not in the orbit of such a word (this can be proved using [13, Proposition 4.19]).

Theorems 4.9 and 4.11 treat curves, on the punctured torus, of self-intersection number one less than the maximum for their length; we do not have similar formulas for the distribution of other self-intersection numbers among curves of a given length. Here is some numerical evidence, computed using the algorithm given in [9]; see also 
[6]; the Java program can be found at [8]. This evidence was in fact the motivation for the research presented here.

Computational Theorem 1.7. The number of distinct primitive free homotopy classes with a given number of self-intersections, corresponding to primitive reduced cyclic words of a given length appears, for length up to 12, in Table 1. (If one entry of a row of Table 1 is 0 then all the entries to its right are also 0.)

\begin{tabular}{|c|r|r|r|r|r|r|r|r|r|r|r|}
\hline length $\backslash$ SI & 0 & 1 & 2 & 3 & 4 & 5 & 6 & 7 & 8 & 9 & 10 \\
\hline 1 & $\mathbf{4}$ & 0 & 0 & 0 & 0 & 0 & 0 & 0 & 0 & 0 & 0 \\
\hline 2 & $\mathbf{4}$ & 0 & 0 & 0 & 0 & 0 & 0 & 0 & 0 & 0 & 0 \\
\hline 3 & $\mathbf{8}$ & 0 & 0 & 0 & 0 & 0 & 0 & 0 & 0 & 0 & 0 \\
\hline 4 & 10 & $\mathbf{8}$ & 0 & 0 & 0 & 0 & 0 & 0 & 0 & 0 & 0 \\
\hline 5 & 16 & 8 & $\mathbf{2 4}$ & 0 & 0 & 0 & 0 & 0 & 0 & 0 & 0 \\
\hline 6 & 8 & 16 & 32 & 40 & $\mathbf{2 0}$ & 0 & 0 & 0 & 0 & 0 & 0 \\
\hline 7 & 24 & 16 & 32 & 48 & 112 & 24 & $\mathbf{5 6}$ & 0 & 0 & 0 & 0 \\
\hline 8 & 16 & 24 & 52 & 76 & 116 & 156 & 136 & 104 & 90 & $\mathbf{4 0}$ & 0 \\
\hline 9 & 24 & 32 & 64 & 120 & 144 & 240 & 384 & 208 & 376 & 136 & 304 \\
\hline 10 & 16 & 32 & 72 & 168 & 272 & 332 & 492 & 628 & 644 & 700 & 700 \\
\hline 11 & 40 & 48 & 80 & 160 & 272 & 584 & 664 & 1200 & 1280 & 1368 & 1608 \\
\hline 12 & 16 & 40 & 104 & 208 & 372 & 660 & 1048 & 1408 & 2044 & 2696 & 3088 \\
\hline
\end{tabular}

\begin{tabular}{|c|r|r|r|r|r|r|r|r|r|}
\hline length \SI & 11 & 12 & 13 & 14 & 15 & 16 & 17 & 18 & 19 \\
\hline 9 & 48 & $\mathbf{1 0 4}$ & 0 & 0 & 0 & 0 & 0 & 0 & 0 \\
\hline 10 & 548 & 464 & 360 & 224 & 160 & $\mathbf{6 8}$ & 0 & 0 & 0 \\
\hline 11 & 1368 & 2048 & 976 & 1704 & 528 & 1072 & 264 & 592 & 80 \\
\hline 12 & 3580 & 3866 & 3792 & 3816 & 3612 & 3272 & 2820 & 2276 & 1808 \\
\hline
\end{tabular}

\begin{tabular}{|c|r|r|r|r|r|r|r|}
\hline length \SI & 20 & 21 & 22 & 23 & 24 & 25 & 26 \\
\hline 11 & $\mathbf{1 6 8}$ & 0 & 0 & 0 & 0 & 0 & 0 \\
\hline 12 & 1308 & 960 & 680 & 392 & 250 & $\mathbf{1 0 4}$ & 0 \\
\hline
\end{tabular}

Table 1: The $i, j$ entry in this table is the number of distinct reduced primitive cyclic words of length $i$ with exactly $j$ self-intersections, up to the maximum possible selfintersection number for each length. Bold-face numbers and their location correspond to Theorems 1.1 and [1.2, italic numbers to Theorem 4.11,

Computational Theorem 1.8. Let $k \in\{1,2, \ldots, 25\}$ and let $K$ be the set of all cyclic reduced words $v$ corresponding to primitive free homotopy classes of curves on the punctured torus, with $\mathrm{SI}(v) \geq k$. If $w$ is a word in $K$ with minimal length then the following statements hold:

(1) The length of $w$ is equal to the smallest integer larger than or equal to $2 \sqrt{k}+2$. 
(2) $\operatorname{SI}(w)=k$.

\subsection{Related results}

For a reduced cyclic word $w$ written in the symbols $\{a, A, b, B\}$, let $\alpha(w)$ and $\beta(w)$ denote the total number of occurrences of $a, A$ and of $b, B$, respectively. Andrew Blood 3. gives a simple construction of a representative curve which has at most $(\alpha(w)-$ 1) $(\beta(w)-1)$ intersections; he also finds some of the words whose representative curves require this number of self-intersections, namely those of the form $a^{\alpha} b^{\beta}$. Together these two discoveries constitute a different proof of the first part of our Theorem 1.1 (compare Theorem 1.10). In addition, Frank Chemotti and Andrea Rau [7] give elementary proofs of parts (2), (3) and (4) of our Proposition 3.6. This unpublished work only came to our attention during the final editing of this paper.

Birman and Series [2] give an algorithm to decide whether a simple representative exists for a reduced cyclic word in the generators of the fundamental group of a surface with boundary. These ideas are extended by Cohen and Lustig [9] (see also [6] and [21]), who give an algorithm to compute the self-intersection of a reduced cyclic word. The program to compute Table 1.7 is based on these algorithms.

From the geometric point of view, the punctured torus has been studied as a manifold with boundary: the complement in $S^{1} \times S^{1}$ of an open disc. This manifold admits a complete hyperbolic metric for which the boundary circle is a geodesic. Since every free homotopy class contains exactly one geodesic representative, and since a primitive geodesic cannot have excess intersections [10], the results in this section translate into results about counting geodesics on that Riemann surface.

(1) It follows from Cohen and Lustig [9, Main Theorem] (see also [6, Proposition 2.9 and Remark 3.10]) that for any surface $S$ with non-empty boundary and negative Euler characteristic, $\operatorname{SI}(w) \leq L(L-1) / 2$ (using our notation) for $w$ a primitive word of length $L$ in the generators (and their inverses) of the fundamental group of $S$. For the torus with one boundary component, the special case examined here, our upper bound (Theorem 1.2) is lower.

For the torus with one geodesic boundary, once a pair of free generators is chosen for the fundamental group then any hyperbolic metric, restricted to closed geodesics, is quasi-isometric to the word-length metric. This is a special case of the Švarc-Milnor Lemma [16], 4]. Hence we can refer to word-length as combinatorial length.

(2) Lalley [12, Theorem 1] proved that on a compact, hyperbolic, closed surface most closed geodesics of length approximately $\ell$ have about $C \ell^{2}$ self-intersections for some positive constant $C$ depending on the surface. As a consequence of our Theorem 1.1, in the case of the torus with one geodesic boundary component, for 
each hyperbolic metric there exists a positive constant $C^{\prime}$ such that the number of self-intersection points of every geodesic of length $\ell$ is less than $C^{\prime} \ell^{2}$. (This fact also admits an elementary proof, as Lalley pointed out to us). Lalley also studies the distribution on the surface of self-intersection points of a typical geodesic; [12, Theorem 2] may be compared with the patterns in Figure 1.

(3) Basmajian proves in [1, Corollary 1.2] that for any hyperbolic surface there exists an increasing sequence of constants $\left\{M_{k}\right\}, k \geq 1$, tending to infinity so that if $\omega$ is a closed geodesic with self-intersection number $k$, then the hyperbolic length of $\omega$ is greater than $M_{k}$. For the punctured torus and combinatorial length our Corollary 1.3 gives explicit values for $M_{k}$, and our bounds are sharp.

In view of the quasi-isometry between combinatorial and hyperbolic length for the torus with one boundary component the numbers in Computational Theorem 1.7 are concordant with numbers or estimates from several other lines of research:

(1) It is known that for any hyperbolic surface the total number of primitive closed geodesics of length at most $L$ is asymptotic to $e^{h L} / L$ ( $h$ is the topological entropy of the geodesic flow; see [5] and references therein; similar results hold for the variable curvature case, [11], [14], [18]). On the punctured torus, the number of distinct primitive classes of combinatorial length $L$ at most twelve, i.e. the sum of the numbers in row $L$ of Table 1, appears to be very rapidly asymptotic to $3^{L} / L$.

(2) The numbers in the first column of Table 1, giving the number of simple classes for a given length, can be compared with the results of McShane and Rivin 15] for the punctured torus and Mirzakhani [17] for a general surface of negative Euler characteristic (see also [19] for historical brackground). Mirzakhani, McShane and Rivin prove that the number of simple closed geodesics of hyperbolic length at most $L$ grows as a quadratic polynomial in $L$ (contrast with Theorem 1.2, where the number of maximal curves of length exactly $L$ grows quadratically with $L$ ). For the range of Table 1, we have data consistent with these: the number of simple curves of length exactly $2 n+1, n \geq 1$, appears to grow more or less linearly with $n$; for $2 n+1$ a prime, it is exactly $8 n$.

(3) For $L$ even, the numbers in the second column of Table 1 grow as $4(L-2)$. This is consistent with Rivin's [20] determination that the number of single-selfintersection geodesics of length at most $L$ grows quadratically with $L$.

(4) For a closed surface $S$, Basmajian proves in [1, Proposition 1.3] that there are constants $N_{k}$ (depending on the genus of $S$ ) such that the shortest geodesic on $S$ with at least $k$ intersection points has length bounded above by $N_{k}$. This generalizes Buser's proof [5] that $N_{1}=1$. Computational Theorem [1.8 gives $N_{k}$ an explicit value for curves of combinatorial length less than 13 on the punctured torus. 


\subsection{Sketch of proof}

The method of proof in this paper keeps track of three integer parameters of a reduced cyclic word $w$ in the alphabet $a, b, A, B$ : along with $\alpha(w)$ and $\beta(w)$ (see Section 1.1) there is $h(w)$, the total number of block-pairs in $w$; these are defined as follows:

Definition 1.9. A reduced cyclic word $w$ is either a pure power or there exist pairs of positive integers $j_{1}, k_{1}, \ldots j_{n}, k_{n}, n \geq 1$, such that $w=\left\langle r_{1}^{j_{1}} s_{1}^{k_{1}} r_{2}^{j_{2}} s_{2}^{k_{2}} \ldots r_{n}^{j_{n}} s_{n}^{k_{n}}\right\rangle$, where $r \in\{a, A\}$ and $s \in\{b, B\}$. Each of the $r_{i}^{j_{i}} s_{i}^{k_{i}}$ occurring in this expression is a block-pair; the number of block-pairs of $w$ is defined to be $n$ in the second case, and zero in the first.

The main theorem in this paper is Theorem 1.10; it will be proved in Section 4 .

Theorem 1.10. For the punctured torus, let $w$ be the reduced cyclic word corresponding to a free homotopy class of curves with a positive number $h$ of block-pairs. If $h=1$ then $\operatorname{SI}(w)=(\alpha(w)-1)(\beta(w)-1)$. If $h \geq 2$,

$$
\mathrm{SI}(w) \leq(\alpha(w)-1)(\beta(w)-1)-h+2 .
$$

The words $w$ realizing the maximal self-intersection for non-pure-power words with given $\alpha$ and $\beta$ (that is, $\operatorname{SI}(w)=(\alpha(w)-1)(\beta(w)-1)$ ) have one of the following forms,

(2) $\left\langle r^{i} s^{j} r^{k} S^{l}\right\rangle$, all $i, j, k, l>0$, where $r \in\{a, A\}$ (and then $i+k=\alpha(w)$ ) and $s \in\{b, B\}$ (and then $j+l=\beta(w)$ ), or vice-versa.

This theorem has two immediate corollaries:

Corollary 1.11. Let $w$ be the reduced cyclic word corresponding to a primitive free homotopy class of curves on the punctured torus. Then

$$
\mathrm{SI}(w) \leq(\alpha(w)-1)(\beta(w)-1)
$$

Corollary 1.12. Among primitive words those of maximal self-intersection number for their $\alpha$ and $\beta$ values, i.e. with $\operatorname{SI}(w)=(\alpha(w)-1)(\beta(w)-1)$, have one of the following forms:

(1) $\langle r\rangle, r \in\{a, b, A, B\}$,

(2) $\left\langle r^{i} s^{j}\right\rangle, r \in\{a, A\}, s \in\{b, B\}$; here $\alpha(w)=i>0, \beta(w)=j>0$.

(3) $\left\langle r^{i} s^{j} r^{k} S^{l}\right\rangle$, all $i, j, k, l>0$, where $r \in\{a, A\}$ (and then $i+k=\alpha(w)$ ) and $s \in\{b, B\}$ (and then $j+l=\beta(w)$ ), or vice-versa. 
Remark 1.13. Since $\alpha(w)+\beta(w)=L$, the length of $w$, an elementary calculation leads from Corollary 1.11 and Corollary 1.12 to Theorem 1.1 .

The next three sections carry the proof of Theorem 1.10, The strategy is to show that only words of the types listed in the statement of the theorem, i.e. $\left\langle r^{i} s^{j}\right\rangle$ and $\left\langle r^{i} s^{j} r^{k} S^{l}\right\rangle, \quad r \in\{a, A\}, s \in\{b, B\}$, or vice-versa, can have maximum self-intersection number for their length; this will be done by exhibiting, for any word which is not of these types, another word of the same length and with strictly larger self-intersection number. For most words $w$, "cross-corner surgery" (defined below) will produce a $w^{\prime}$ with the same $\alpha$ and $\beta$ values (and so of the same length), with $\operatorname{SI}\left(w^{\prime}\right)>\operatorname{SI}(w)$ and with $h\left(w^{\prime}\right)<h(w)$ For certain words with two, three or four blocks, not candidates for surgery, the self-intersection number will be computed explicitly by counting "linked pairs" of subwords (definition below) and determining that it is indeed smaller than the self-intersection number of a word of the same length but of one of the two listed types (whose self-intersection numbers are also computed by counting linked pairs).

This work benefited from discussions with Ara Basmajian, Joel Hass, Stephen Lalley, Igor Rivin and Dennis Sullivan.

\section{Cross-corner surgery}

\subsection{Preliminaries}

Here, let $M$ represent the punctured torus as a topological space. The choice of generators $(a, b)$ for $\pi_{1} M$ naturally implies a fundamental polygon from which $M$ may be reconstructed by edge-identification. Namely, we can choose, as representative cycles for the homological duals $a^{*}, b^{*} \in H_{1}(M / \partial M)$, two disjoint, connected arcs beginning and ending in $\partial M$; slicing $M$ along these arcs gives a simply-connected polygon which can serve as fundamental domain (for the action of $\pi_{1}(M)$ on the universal cover); for our purposes we will label $a$ the edge keeping the orientation of $a^{*}$, and $A$ its opposite edge with the opposite orientation (see Figure 2); similarly for $b$ and $B$. Lifting a curve in $M$ to this fundamental polygon means representing the curve as a set of arcs-withidentifications; each of these curve segments leads from one of the edges $a, b, A, B$ to another; the orientation of the curve defines a cyclic word in the four symbols: one records the positive intersections as they occur. By construction, this word represents the free homotopy class of the curve under consideration.

A curve segment is a transversal if it joins opposite edges of the fundamental domain, and a corner otherwise. Transversals correspond to consecutive $a a, A A, b b, B B$ in the word; other combinations give corners. Two corners are opposite if they are diagonally opposed. Thus $a b, b a$ and $a b, A B$ correspond to diagonally opposed corners; $a b$ and $b a$ have the same orientation whereas $a b$ and $A B$ have reversed orientations. In Figure 2 the curve $\langle b a B B A b a\rangle$ has two $b a$ corner segments diagonally opposed to an $a b$ (same 


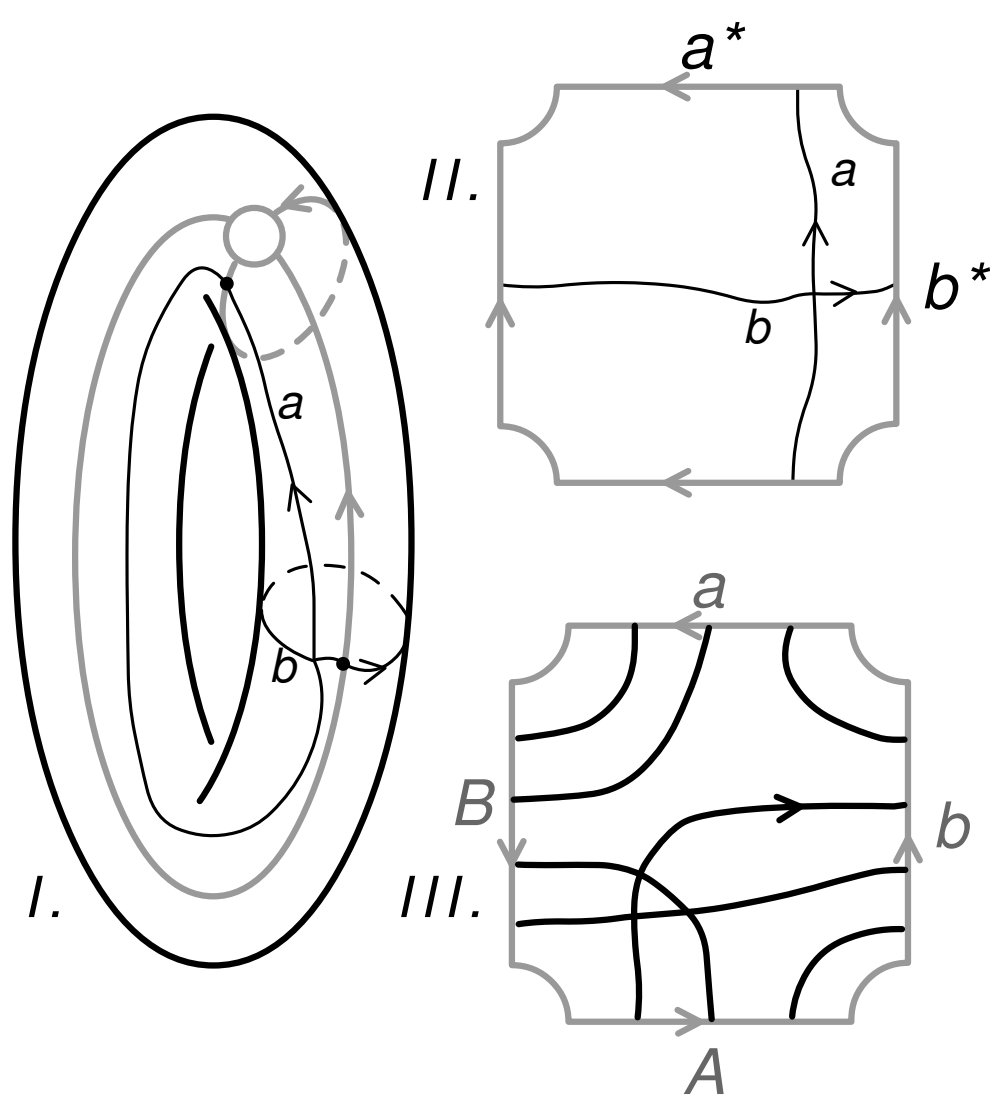

Figure 2: The punctured torus as a polygon with identifications. I, II. The generators $a, b$ and their inverses $A, B$ can be identified by their intersections with the dual cycles $a *, b *$, which appear among the edges of the fundamental polygon. III. When an oriented curve has been lifted to the fundamental polygon, the cyclic word corresponding to its free homotopy class can can be obained by choosing a starting point and recording in sequence the edges it crosses, reading their names from inside the polygon. The lifted curve $\langle b a B B A b a\rangle$ with self-intersection number 3 , is shown as an example. 
orientation) and a $B A$ (reversed orientation); one $a B$ corner diagonally opposed to opposed to an $a b$ (same orientation) and a $B A$ (reversed orientation); one $a B$ corner diagonally opposed to an $A b$ corner (reversed orientations) and one $B B$ transversal.

A curve with only transversal self-intersections, and with the smallest number of self-intersections for its homotopy class (multiple points count with multiplicity: a multiple intersection of $n$ small arcs counts as $\left(\begin{array}{l}n \\ 2\end{array}\right)$ intersections) is said to be tight (compare [22]: "taut").

Two-component multi-words $\left[w, w^{\prime}\right]$ enter into the surgery process. We define the intersection number $\operatorname{IN}\left(w, w^{\prime}\right)$ of two reduced cyclic words $w, w^{\prime}$ to be the minimum number of intersections between a general-position curve representing $w$ and one representing $w^{\prime}$. The self-intersection number of the multi-word $\left[w, w^{\prime}\right]$ is then $\mathrm{SI}\left(\left[w, w^{\prime}\right]\right)=\mathrm{SI}(w)+\mathrm{SI}\left(w^{\prime}\right)+\mathrm{IN}\left(w, w^{\prime}\right)$, and a pair of curves with that smallest number of self-intersections is also said to be tight. We also extend the $\alpha$ and $\beta$ notation to multiwords: $\left.\left.\alpha(] w^{\prime}, w^{\prime \prime}\right]\right)$ is the total number of occurrences of $a$ or $A$ in $w$ and $w^{\prime}$; $\beta\left(\left[w^{\prime}, w^{\prime \prime}\right]\right)$ the total number of occurrences of $b$ or $B$.

\subsection{The surgery}

Whenever a cyclic word $w$ contains a pair of opposite corners, it may be cut in two places, once in the middle of each of the corners, to give two linear words. These two linear words may be reassembled (the corners themselves are reassembled into transversals) into either a new word $w^{\prime}$ or a new multi-word $\left[w^{\prime}, w^{\prime \prime}\right]$ (according to the relative orientation of the corners); if a multi-word $\left[v^{\prime}, v^{\prime \prime}\right]$ contains a pair of opposite corners, one in each component, the two corners may be cut and reassembled into two transversals yielding a new single word $v$.

For a picture of the surgery on a curve, see Figure 3, in terms of the words, the cutting and reassembly take one of the following forms:

$$
\begin{aligned}
\langle\mathrm{x} r|s \mathrm{y} s| r \mathrm{z}\rangle & \rightarrow[\langle\mathrm{x} r \mid r \mathrm{z}\rangle,\langle s \mathrm{y} s \mid\rangle] \\
\langle\mathrm{x} r|s \mathrm{y} R| S \mathrm{z}\rangle & \rightarrow\langle\mathrm{X} r|r \mathrm{Y} S| S \mathrm{z}\rangle \\
{[\langle\mathrm{x} r \mid s \mathrm{y}\rangle,\langle\mathrm{z} s \mid r \mathrm{w}\rangle] } & \rightarrow\langle\mathrm{x} r|r \mathrm{wz} s| s \mathrm{y}\rangle \\
\{\langle\mathrm{x} r \mid s \mathrm{y}\rangle,\langle\mathrm{z} R \mid S \mathrm{w}\rangle\} & \rightarrow\langle\mathrm{x} r|r \mathrm{ZX} s| s \mathrm{y}\rangle
\end{aligned}
$$

where $\mathrm{x}, \mathrm{y}, \mathrm{z}, \mathrm{w}$ are arbitrary (linear) subwords, and $R=r^{-1}, S=s^{-1}, \mathrm{X}=\mathrm{x}^{-1}$, etc.

Definition 2.1. This cutting and reassembly are called cross-corner surgery on the word $w$ or the multi-word $\left[v^{\prime}, v^{\prime \prime}\right]$.

It seems natural that transversals should contribute, more than corners, to the selfintersection number of a curve. Proposition 2.2 makes this quantitative by showing that cross-corner surgery, which eliminates two corners and adds two transversals, always increases the self-intersection number by at least one. 
Proposition 2.2. (1) If a word $w$ contains a pair of opposite corners with reversed orientation then cross-corner surgery will produce a new word $w^{\prime}$, with $\alpha\left(w^{\prime}\right)=$ $\alpha(w), \beta\left(w^{\prime}\right)=\beta(w)$, with one less block-pair, and with $\operatorname{SI}\left(w^{\prime}\right) \geq \operatorname{SI}(w)+1$.

(2) If a word $w$ contains a pair of opposite corners with the same orientation then cross-corner surgery will produce a multi-word $\left[w^{\prime}, w^{\prime \prime}\right]$, with $\alpha\left(\left[w^{\prime}, w^{\prime \prime}\right]\right)=\alpha(w)$, $\beta\left(\left[w^{\prime}, w^{\prime \prime}\right]\right)=\beta(w)$, with one less block-pair, and with $\mathrm{SI}\left(\left[w^{\prime}, w^{\prime \prime}\right]\right) \geq \operatorname{SI}(w)+1$.

(3) If a multi-word $\left[v^{\prime}, v^{\prime \prime}\right]$ contains a pair of opposite corners, one in each component, irrespective of orientation, then cross-corner surgery will produce a single word $v$ with $\alpha(v)=\alpha\left(\left[v^{\prime}, v^{\prime \prime}\right]\right), \beta(v)=\beta\left(\left[v^{\prime}, v^{\prime \prime}\right]\right)$, with one less block-pair, and with $\mathrm{SI}(v) \geq \mathrm{SI}\left(\left[v^{\prime}, v^{\prime \prime}\right]\right)+1$.

This proposition is stated in terms of words, but its proof, given in the next subsection, works by examining curves representing the words before and after surgery; we first must fix a topological procedure for carrying out cross-corner surgery on a curve. Specifically, given a tight curve, or a tight pair of curves, representing the candidates $w$ or $\left[v^{\prime}, v^{\prime \prime}\right]$ for cross-corner surgery, we need to establish a systematic way of generating curves representing the result $w^{\prime},\left[w^{\prime}, w^{\prime \prime}\right]$ or $v$ of the surgery. We do this as follows:

Definition 2.3. Cross-corner surgery on curves. Suppose $r \mid s$ and $s \mid r$ or $R \mid S$ are the loci (that is, two diagonally opposite corners) in the word $w$ (or the multi-word $\left.\left[v^{\prime}, v^{\prime \prime}\right]\right)$ chosen for surgery, and let $K$ and $L$ be the corresponding corners in a tight representative (see Figure 3).

- Preparation for the surgery. If the extension of any corner segment of the same type as $K$ (i.e. corresponding to the same letter sequence $r s$ or to the inverse sequence $S R$ ) intersects the extension of $K$ in either direction before diverging, the curve is prepared for surgery by a homotopy sliding that (necessarily single) intersection onto the segment $K$ itself. This deformation may be carried out by a sequence of Reidemeister-type-III moves without changing the total number of intersections (see Figure 3, I and II). A similar operation is carried out on the corner $L$.

- Cutting and Sewing. Corresponding to the word permutation, corners $K$ and $L$ are removed and replaced by transversals $U$ and $V$. More precisely, a line is drawn from a point on $K$ to a point on $L$, in general position with respect to the rest of the curve, and cutting any segment no more than once; that line is expanded into an $\mathcal{X}$-junction: $U$ routes the right edge of $K$ to the left edge of $L$, and vice-versa for $V$. 

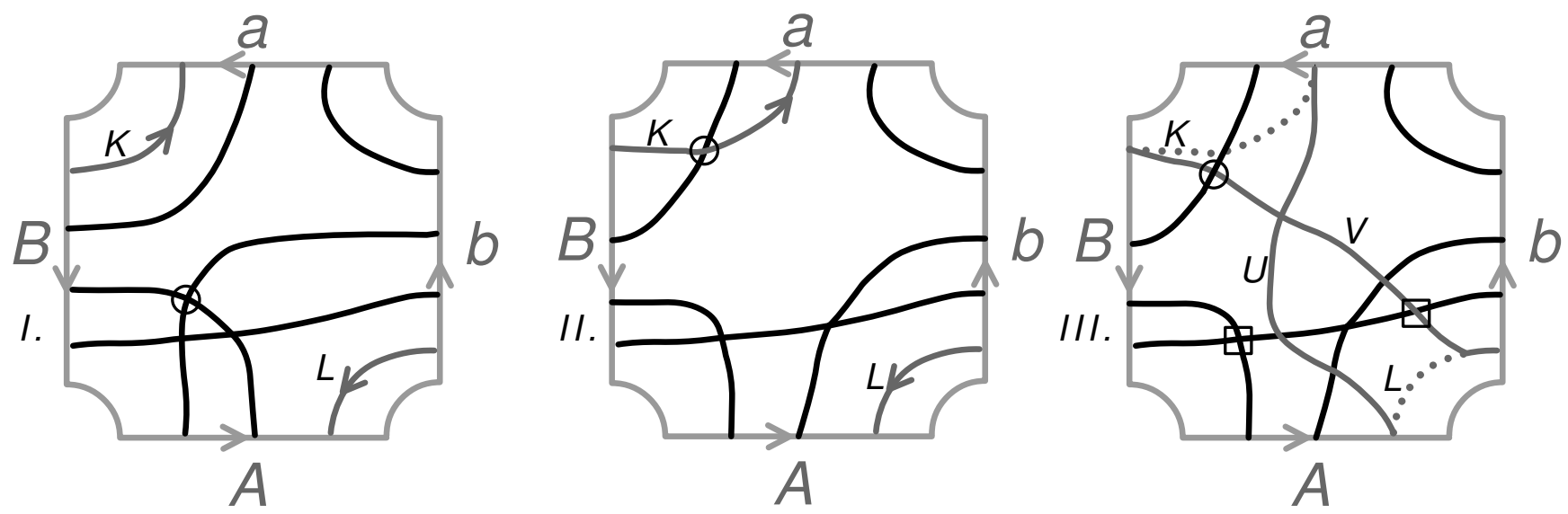

Figure 3: The cross-corner surgery $\langle b a B B|A b| a\rangle \rightarrow\langle b a B B|B a| a\rangle$ as carried out on a tight representative curve. I. The corner $K$ corresponds to $b \mid a ; L$ corresponds to $B \mid A$. Note that the extension of $K$ intersects that of one of its parallel corners (circled intersection). II. Before surgery, that intersection is "pushed," using a Rademeistertype-III move, into the center of the surgery. III. $K$ and $L$ are excised, $U$ and $V$ sewn in. The circled intersection migrates to an intersection with $V$. The intersection of $V$ with the original $B B$ spans a bigon with one of the original vertices (squared intersections $)$. $\mathrm{SI}(\langle b a B B B a a\rangle)=6$.

\subsection{Proof of Proposition 2.2}

We will obtain a lower bound on the increase in self-intersection number by counting the vertices added and those possibly annihilated by the surgery. Annihilation occurs through the creation of a bigon: an immersed planar polygon with two vertices, and two edges with disjoint preimages (a "singular 2-gon" in [10]); the bigon defines a homotopy of the curve leading to the disappearance of its two vertices. An intersection will be called stable if it is not the vertex of a bigon; a curve is tight if all its self-intersections are stable [10.

Lemma 2.4. Cross-corner surgery does not create any bigons spanned by a pair of pre-surgery vertices.

Proof. Since the initial curve is tight, the only way a pair $x, y$ of curve-portions starting from a pre-surgery ("old") vertex $P$ can lead to a bigon with another old vertex is if one of those curve-portions (say, $x$ ) contains one of the new segments $U$ or $V$, say $U$. Suppose the other one, i.e. $y$, enters inside the corner ( $L$ in Figure 4). Then (Figure 4 , I) as $y$ follows $x$ across the frame $y$ must intersect $L$ in an old vertex $P^{\prime}$ cancelling $P$, contradicting tightness of the original curve. So $y$ must enter outside $L$; then running parallel to $U$ across the frame it must intersect the opposite corner $K$ in an old vertex $Q$ (Figure 4, II). Now if $x$ and $y$ meet in an old vertex $Q^{\prime}$ so as to form a bigon cancelling 

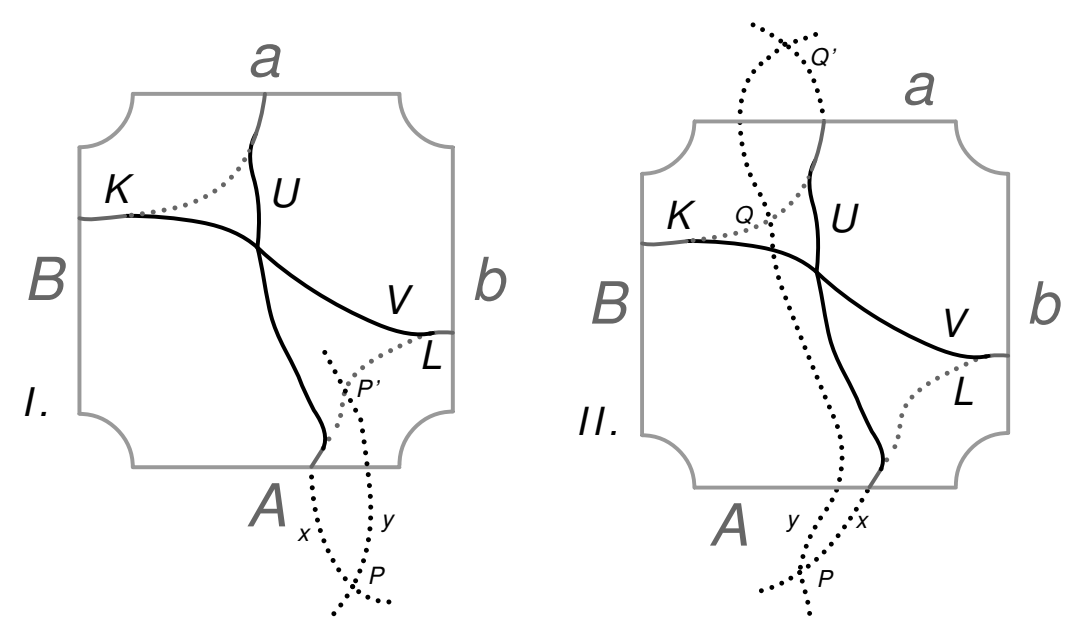

Figure 4: Cross-corner surgery cannot produce a bigon linking two pre-surgery vertices.

$P$, then $Q^{\prime}$ and $Q$ will span an old-vertex bigon. By tightness, this will require another use of the new segments. Since each of $U$ and $V$ can only be used once by each of $x$ and $y$, after at most four passes through the frame all the possibilities will be exhausted; no such bigon can exist.

Proof of Proposition 2.2 The curve surgery described in Definition 2.3 yields one word if it is applied to a word that contains a pair of opposite corners with reversed orientation, a multi-word if it is applied to a word that contains a pair of opposite corners with the same orientation and a single word if it is applied to a multi-word that contains a pair of opposite corners, one in each component, irrespective of orientation. Thus, to prove (1), (2) and (3) it is enough to prove that in a cross-corner surgery, the number of new vertices minus the number of vertices cancelled by new bigons is greater than or equal to one. We start by classifying the new vertices introduced by the surgery and the possible bigons in which they may participate.

\section{Vertices:}

The surgery creates three types of new vertices, shown as black, grey and white in Figure 5, as follows.

(1) (black) Stable intersections between $U$ and horizontal transversals (i.e segments corresponding to $b b$ or $B B$ in the initial word $w$ ), between $V$ and vertical transversals, and (bullseye) the stable intersection between $U$ and $V$.

(2) (grey) Intersections between $U$ and other vertical transversals, and between $V$ and other horizontal transversals. These are potentially vertices of bigons.

(3) (white) Intersections between $U, V$ and remaining corner segments. In Figure 5 only those of type $a b$ or $B A$ are shown; there is typically another family in the 


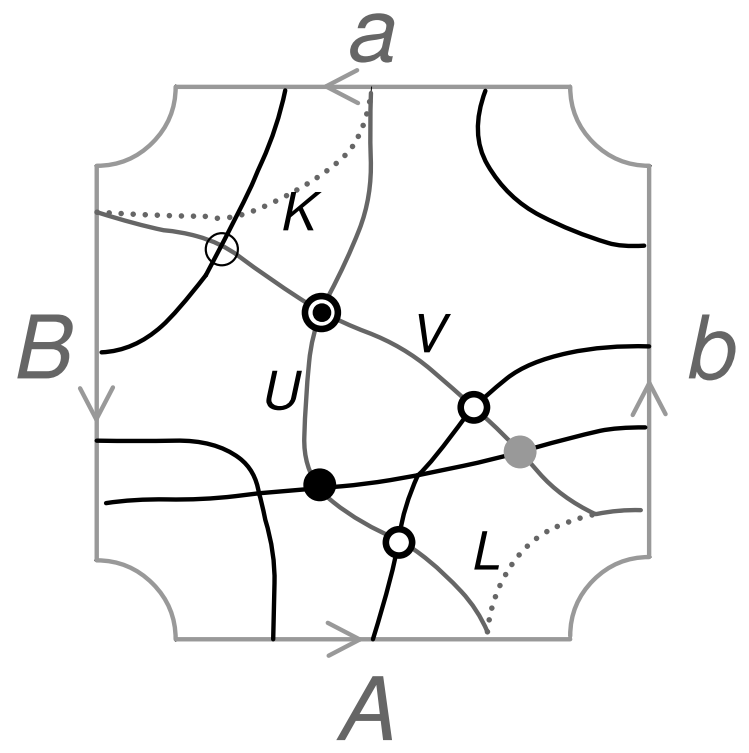

Figure 5: The new vertices created by a cross-corner surgery.

opposite corner corresponding to types $b a$ or $A B$. These are also potentially vertices of bigons.

(4) In addition, the circled vertices in Figure 5 are those inherited by the new curve from the old. These correspond to the intersections between $K$ or $L$ with other corners of the same type; such a corner is labeled $J$ in Figure 6. Focussing on $K$, let us label $x$ and $y$ the two ends of the segment $K$, and by $w$ the intersection point of the new segments $U$ and $V$. The segment $K$ and the broken curve $u w v$ are fixed-endpoint homotopic; it follows that for any original segment having exactly one endpoint between $u$ and $v$ (e.g. $J$ ) the intersection with $K$ will migrate to an intersection with $U$ or $V$ during that homotopy (with $V$ if the outside end of $J$ is on the $B$ side -as in Figure [6- and with $U$ if it is on the $a$ side).

\section{Bigons:}

The only bigons that need to be examined are those where one of the spanning vertices is an old vertex or a type- 4 vertex; because if two new vertices form a bigon and cancel, that does not affect the inequality we need to prove. So, letting 1, 2, 3, and 4 represent vertices so labeled above, letting $x, x^{\prime}$ represent self-intersections of the original curve, and keeping in mind that type- 1 vertices are stable, and that bigons of type $\left(x, x^{\prime}\right)$ cannot occur (Lemma 2.4), we need only examine bigons of type $(2, x)$, $(2,4),(3, x),(3,4),(4, x)$, and $(4,4)$.

(i) $(4, x)$ and $(4,4)$. A vertex of type 4 can span a bigon in only one of its quadrants; but in that quadrant a bigon would imply a bigon with the old vertex from which 


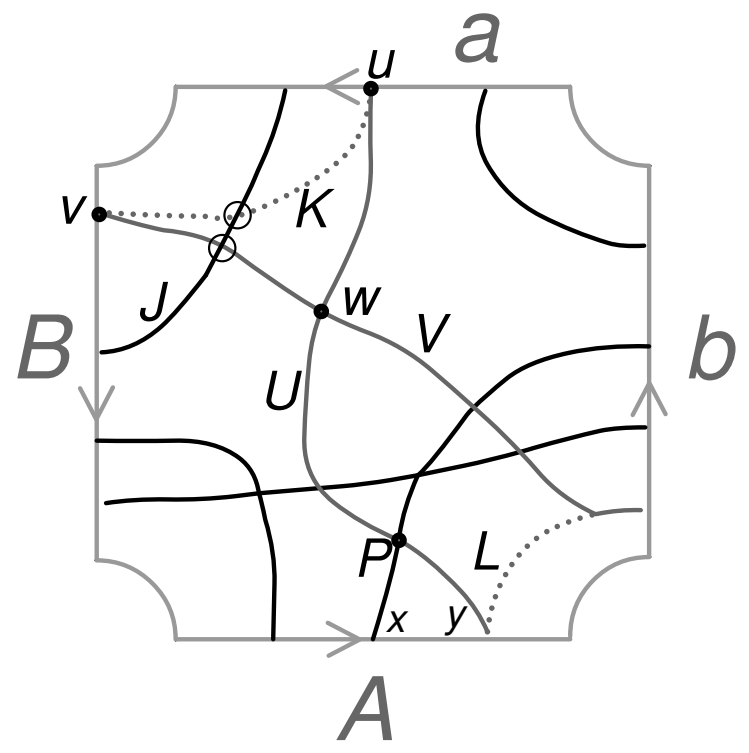

Figure 6: Vertices inherited by new curve from old; $P$ is an example of a type-3 vertex.

the type- 4 vertex was inherited; so a $(4, x)$ would imply an $\left(x^{\prime}, x\right)$, and a $(4,4)$ would imply a $(4, x)$; so neither $(4,4)$ nor $(4, x)$ can occur.

(ii) $(2, x)$ and $(2,4)$. A type-2 vertex $y$ may span a bigon with an old vertex $x$; the type-2 vertex is either the intersection of $U$ with another vertical transversal, or $V$ with another horizontal. In the first case (the second case is similar), that vertical transversal must also intersect $V$, creating a new (type-1) stable intersection $z$. In total we will have added two vertices $(y$ and $z$ ), and lost two vertices ( $y$ and $x)$ to a bigon. The inequality is not affected.

Since, arguing as in (i), a $(2,4)$ bigon would imply a $(2, x)$ bigon, the loss of the 4 would be balanced by the gain of the corresponding new type-1 vertex, and again the inequality would not be affected.

(iii) $(3, x)$ and $(3,4)$. Figure 6 shows a typical type-3 vertex $P$. It can only span a bigon in one quadrant; label $x$ and $y$ the two segments issuing from $P$ in that direction. Because of the way the curve is prepared for surgery, $x$ and $y$ cannot be continued with old segments to form a bigon cancelling $P$. We need to discuss the possibility that after surgery their extensions could incorporate $U$ or $V$ or both and then form such a bigon. This $P, x$ and $y$ exactly match the notation of Lemma 2.4 and the proof of that lemma applies here as well: no such bigon can exist. Since a $(3,4)$ bigon would imply a $(3, x)$ bigon, no type- $(3,4)$ bigons can exist either.

In summary, cross-corner surgery generates one special stable vertex (the intersec- 
tion of $U$ and $V$ ) plus other new vertices of types 1,2,3, and displaced vertices of type 4 . Vertices of type 1 are stable. Some of the vertices of type 2 and 3 form bigons with each other and cancel out. Vertices of type 3 and 4 cannot form bigons with pre-surgery vertices, and any old or type- 4 vertex cancelled by a type- 2 vertex can be replaced in the count by the corresponding type- 1 vertex. It follows that cross-corner surgery increases the self intersection number by at least one.

\section{Linked pairs}

Ultimately the calculation of $\operatorname{SI}(w)$ or $\operatorname{IN}\left(\left[w^{\prime}, w^{\prime \prime}\right]\right)$ can be made directly from $w$ or $\left[w^{\prime}, w^{\prime \prime}\right]$, by counting linked pairs. In this section we give a simplified definition appropriate for the punctured torus, we list two theorems from [6] giving the correspondence between linked pairs and intersection points, and we summarize explicit calculations of intersection and self-intersection numbers for certain families of words with a small number of block-pairs. Linked pairs have also been defined and studied by Cohen and Lustig [9].

Notation 3.1. From now on, we will use the symbols $p, q, r, s, p_{1}, q_{1}$, etc. to represent letters from the alphabet $a, b, A, B$, with $P=p^{-1}$, etc. The symbols $v, w, v^{\prime}, w^{\prime}$, etc will represent cyclic words in that alphabet, e.g. $w=\langle a b b a B\rangle=\langle a B a b b\rangle$. Sans-serif symbols $\mathrm{u}, \mathrm{v}$, y will represent linear words in the alphabet $\{a, b, A, B\}$ with $\mathrm{r}, \mathrm{s}, \mathrm{R}, \mathrm{S}$ representing homogeneous blocks of letters $r r \ldots r, s s \ldots s, R R \ldots R, S S \ldots S$ respectively. As before, $\mathrm{V}=\mathrm{v}^{-1}, \mathrm{R}=\mathrm{r}^{-1}$, etc.

Orientation: For these purposes we identify the boundary of our fundamental domain with a clock face, with $a, b, A, B$ at 3, 6, 9 and 12 o'clock. Given six letters $p, q, r, p^{\prime}, q^{\prime}, r^{\prime}$ from the alphabet $a, b, A, B$, we say that the triples $p, q, r$ and $p^{\prime}, q^{\prime}, r^{\prime}$ are similarly oriented if the arcs $p q r$ and $p^{\prime} q^{\prime} r^{\prime}$ have the same orientation on the clock face. This implies that the three points in each triple are distinct.

Definition 3.2. Let $w$ (resp. $\left[w^{\prime}, w^{\prime \prime}\right]$ ) be a primitive reduced cyclic word (resp. a multi-word with primitive reduced cyclic components), corresponding to a free homotopy class (resp. a pair of free homotopy classes) of curves on the punctured torus.

Let $\mathrm{u}^{\prime}$ and $\mathrm{u}^{\prime \prime}$ be two possibly overlapping but distinct linear subwords, both of the same length $\geq 2$, of $w$ or of $\left[w^{\prime}, w^{\prime \prime}\right]$ (in that case let $\mathrm{u}^{\prime} \subset w^{\prime}$, and let $\mathrm{u}^{\prime \prime} \subset w^{\prime \prime}$ ). The pair of words $\left\{\mathbf{u}^{\prime}, \mathrm{u}^{\prime \prime}\right\}$ is a linked pair if one of the following criteria is satisfied (see Figure 7).

I. $\left[\mathbf{u}^{\prime}, \mathbf{u}^{\prime \prime}\right]$ is one of the following words: $\{a a, b b\},\{a a, B B\},\{A A, b b\},\{A A, B B\}$. 
II. (i) (length 3) $\mathrm{u}^{\prime}=p_{1} r p_{2}, \mathrm{u}^{\prime \prime}=q_{1} r q_{2}$ (same $r$ ) with $P_{1} Q_{1} r$ and $p_{2} q_{2} R$ similarly oriented, or

(ii) (length $n) \mathrm{u}^{\prime}=p_{1} \mathrm{y} p_{2}, \mathrm{u}^{\prime \prime}=q_{1} \mathrm{y} q_{2}, \mathrm{y}=x_{1} \mathrm{v} x_{2}$ (v possibly empty) with $P_{1} Q_{1} x_{1}$ and $p_{2} q_{2} X_{2}$ similarly oriented.

III. (i) (length 3) $\mathbf{u}^{\prime}=p_{1} r p_{2}, \mathbf{u}^{\prime \prime}=q_{1} R q_{2}\left(R=r^{-1}\right)$ with $P_{1} q_{2} r$ and $p_{2} Q_{2} R$ similarly oriented, or

(ii) (length $n$ ) $\mathrm{u}^{\prime}=p_{1} \mathrm{y} p_{2}, \mathrm{u}^{\prime \prime}=q_{1} \mathrm{Y} q_{2}, \mathrm{y}=x_{1} \mathrm{v} x_{2}$ (v possibly empty) with $P_{1} q_{2} x_{1}$ and $p_{2} Q_{1} X_{2}$ similarly oriented.
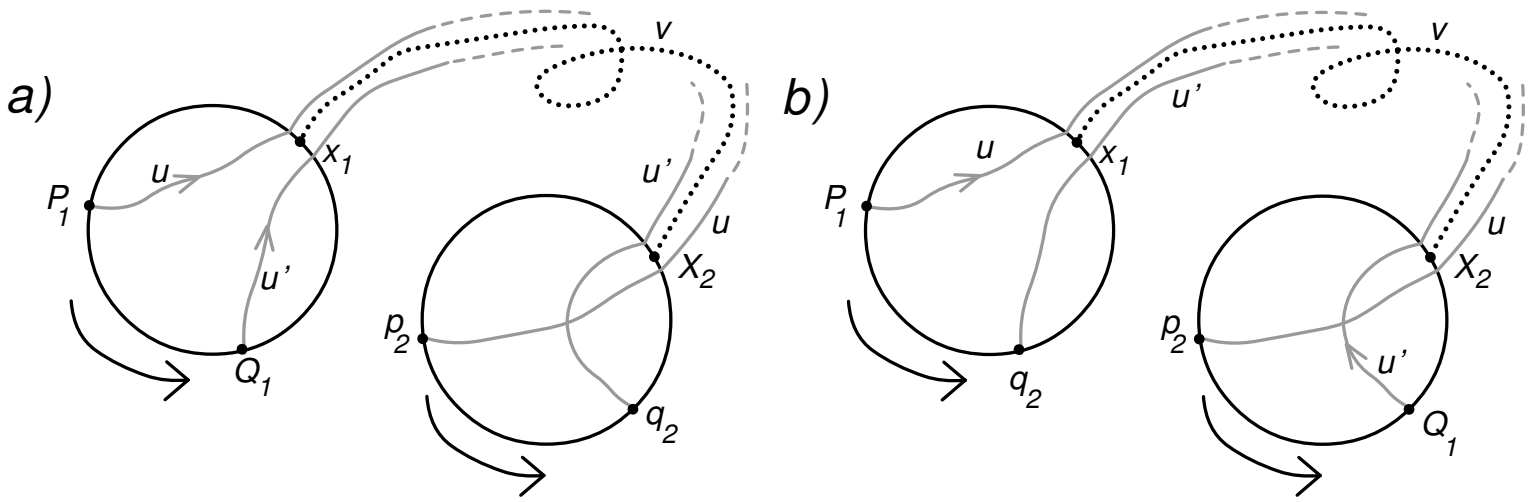

Figure 7: Linked pairs. Here $\mathrm{y}=x_{1} \mathrm{v} x_{2}$. a) The linked pair is $\left(p_{1} \mathrm{y} p_{2}, q_{1} \mathrm{y} q_{2}\right)$. Since the orientations of $\left(P_{1}, Q_{1}, x_{1}\right)$ and $\left(p_{2}, q_{2}, X_{2}\right)$ are the same, the curve segments must intersect. b) The linked pair is $\left(p_{1} \mathrm{y} p_{2}, q_{2} \mathrm{Y} q_{1}\right)$. Since the orientations of $\left(P_{1}, q_{2}, x_{1}\right)$ and $\left(p_{2}, Q_{1}, X_{2}\right)$ are the same, the curve segments must intersect.

Remark 3.3. $\left\{u, u^{\prime}\right\}$ is a linked pair of type (2) if and only if $\left(u, U^{\prime}\right)$ is a linked pair of type (3).

Tables 2, 3] and 4] summarize for future reference the pairing between various subwords of a cyclic word $w$. In these tables an "=" means that the row word and the column word have the same first or last letter (so they cannot form a linked pair); "N" means that there is no end matching but that the pair fails the orientation criterion; "Y" means that the row word and the column word form a linked pair.

The following theorem will be used to compute the self-intersection numbers of certain words and multi-words (see Proposition 3.6 and Appendix A). This theorem is a direct consequence of [6, Theorems 3.9 and 3.10 and Remarks 3.10 and 3.11] and [6, Theorem 3.12 and Remark 3.13].

Theorem 3.4. Let $v$ and $w$ be a cyclic reduced words in the alphabet $\{a, b, A, B\}$. Suppose that $w=\left\langle u^{k}\right\rangle$ is the $k$ th power $(k \geq 0)$ of the primitive reduced cyclic word $u$. 


\begin{tabular}{|l|ccccccc|}
\hline Words & $a^{i+1} b$ & $b a^{i+1}$ & $a^{i+1} B$ & $B a^{i+1}$ & $b a^{i} b$ & $a^{i+2}$ & $B a^{i} B$ \\
\hline$a^{i+1} b$ & $=$ & $\mathrm{N}$ & $=$ & $\mathrm{Y}$ & $=$ & $=$ & $\mathrm{Y}$ \\
$b a^{i+1}$ & $\mathrm{~N}$ & $=$ & $\mathrm{Y}$ & $=$ & $=$ & $=$ & $\mathrm{Y}$ \\
$a^{i+1} B$ & $=$ & $\mathrm{Y}$ & $=$ & $\mathrm{N}$ & $\mathrm{Y}$ & $=$ & $=$ \\
$B a^{i+1}$ & $\mathrm{Y}$ & $=$ & $\mathrm{N}$ & $=$ & $\mathrm{Y}$ & $=$ & $=$ \\
$b a^{i} b$ & $=$ & $=$ & $\mathrm{Y}$ & $\mathrm{Y}$ & $=$ & $\mathrm{Y}$ & $\mathrm{Y}$ \\
$a^{i+2}$ & $=$ & $=$ & $=$ & $=$ & $\mathrm{Y}$ & $=$ & $\mathrm{Y}$ \\
$B a^{i} B$ & $\mathrm{Y}$ & $\mathrm{Y}$ & $=$ & $=$ & $\mathrm{Y}$ & $\mathrm{Y}$ & $=$ \\
\hline
\end{tabular}

Table 2: Linking of pairs of words with $\mathrm{Y}=a^{i}$ (notation from Definition 3.2, $=, \mathrm{Y}, \mathrm{N}$ explained in the text).

\begin{tabular}{|c|cccc|}
\hline Words & $a a^{i} b^{j} b$ & $b a^{i} b^{j} a$ & $b a^{i} b^{j} b$ & $a a^{i} b^{j} a$ \\
\hline$a a^{i} b^{j} b$ & $=$ & $\mathrm{N}$ & $=$ & $=$ \\
$b a^{i} b^{j} a$ & $\mathrm{~N}$ & $=$ & $=$ & $=$ \\
$b a^{i} b^{j} b$ & $=$ & $=$ & $=$ & $\mathrm{Y}$ \\
$a a^{i} b^{j} a$ & $=$ & $=$ & $\mathrm{Y}$ & $=$ \\
\hline
\end{tabular}

Table 3: Linking of pairs of words with $\mathrm{Y}=a^{i} b^{j}$ (notation as in Definition 3.2).

\begin{tabular}{|c|cccc|}
\hline Words & $a a^{i} b^{j} a^{k} b$ & $b a^{i} b^{j} a^{k} a$ & $b a^{i} b^{j} a^{k} b$ & $a a^{i} b^{j} a^{k} a$ \\
\hline$a a^{i} b^{j} a^{k} b$ & $=$ & $\mathrm{N}$ & $=$ & $=$ \\
$b a^{i} b^{j} a^{k} a$ & $\mathrm{~N}$ & $=$ & $=$ & $=$ \\
$b a^{i} b^{j} a^{k} b$ & $=$ & $=$ & $=$ & $\mathrm{Y}$ \\
$a a^{i} b^{j} a^{k} a$ & $=$ & $=$ & $\mathrm{Y}$ & $=$ \\
\hline
\end{tabular}

Table 4: Linking of pairs of words with $\mathrm{Y}=a^{i} b^{j} a^{k}$ (notation as in Definition 3.2). 
(1) If $k=0$, so $w$ is primitive, $\mathrm{SI}(w)$ is equal to the number of linked pairs of $w$, i.e. the cardinality of the set of unordered pairs $\left\{\mathrm{u}, \mathrm{u}^{\prime}\right\}, \mathrm{u}$ and $\mathrm{u}^{\prime}$ linear subwords of $w$, with $\mathrm{u}$ and $\mathrm{u}^{\prime}$ linked as in Definition 3.2.

(2) In general, $\mathrm{SI}(w)$ is less than or equal to $(k-1)$ plus the number of linked pairs of $w$.

(3) $\operatorname{IN}(\{v, w\})$ equals the number of ordered pairs $\left(\mathbf{u}, \mathbf{u}^{\prime}\right)$ for which there exist positive integers $j$ and $k$ such that $\mathrm{u}$ is an occurrence of a subword of $v^{j}$, but not a subword of $v^{j-1}, \mathbf{u}^{\prime}$ is an occurrence of a subword of $w^{k}$, but not a subword of $w^{k-1}$, and $\mathbf{u}$, $\mathrm{u}^{\prime}$ are linked as in Definition 3.2. (See Remark 3.5.)

In this work, only the following simple instances of Theorem 3.4(3) will be necessary.

Remark 3.5. (1) IN $\left(\left\langle a^{i} b^{j}\right\rangle,\left\langle a^{k} B^{l}\right\rangle\right)$ equals the number of ordered pairs $\left(\mathbf{u}, \mathbf{u}^{\prime}\right)$ such that $\mathrm{u}$ is an occurrence of a subword of $\left\langle a^{i} b^{j}\right\rangle, \mathbf{u}^{\prime}$ is an occurrence of a subword of $\left\langle a^{k} B^{l}\right\rangle$ and $\mathrm{u}, \mathrm{u}^{\prime}$ are linked as in Definition 3.2 .

(2) $\operatorname{IN}\left(\left\langle a^{i} b^{j} a^{k} b^{l}\right\rangle,\left\langle a^{m} B^{n}\right\rangle\right)$ equals the number of ordered pairs $\left(\mathbf{u}, \mathbf{u}^{\prime}\right)$ such that $\mathbf{u}$ is an occurrence of a subword of $\left\langle a^{i} b^{j} a^{k} b^{l}\right\rangle, \mathbf{u}^{\prime}$ is an occurrence of a subword of $\left\langle a^{m} B^{n}\right\rangle$ and $\mathrm{u}, \mathrm{u}^{\prime}$ are linked as in Definition 3.2.

This is because if $[v, w]=\left[\left\langle a^{i} b^{j}\right\rangle,\left\langle a^{k} B^{l}\right\rangle\right]$ or $(v, w)=\left[\left\langle a^{i} b^{j} a^{k} b^{l}\right\rangle,\left\langle a^{m} B^{n}\right\rangle\right], J$ and $K$ are non-negative integers, $\mathrm{u}$ is a linear word which is an occurrence of a subword of $v^{J}$ and $w^{K}$ then $\mathrm{u}$ is an occurence of a subword of $v$ and $w$.

In principle the self-intersection number corresponding to any particular word can be ascertained combinatorially by a count of linked pairs. The number of steps in this calculation increases rapidly with the length of the word, but it can be carried out completely for words with a small number of block pairs. The results of these calculations are given in Proposition [3.6, with the work itself presented in Appendix A.

Proposition 3.6. (1) $\operatorname{SI}\left(\left\langle a^{i} b^{j}\right\rangle\right)=(i-1)(j-1)$.

$$
\mathrm{SI}\left(\left\langle a^{i} b^{j} a^{k} b^{l}\right\rangle\right) \begin{cases}\leq(i+k-2)(j+l-2)+1 & \text { if } k=i \text { and } l=j, \\ =(i+k-2)(j+l-2)+|i-k|+|j-l|-1 & \text { otherwise. }\end{cases}
$$

(3) $\operatorname{SI}\left(\left\langle a^{i} b^{j} a^{k} B^{l}\right\rangle\right)=(i+k-1)(j+l-1)$.

(4) $\operatorname{SI}\left(\left\langle a^{i} b^{j} A^{k} B^{l}\right\rangle=(i+k-1)(j+l-1)-1\right.$.

(5) $\operatorname{SI}\left(\left\langle a^{i} b^{j} a^{k} b^{l} a^{m} B^{n}\right\rangle\right)=(i+k+m-1)(j+l+n-1)-2(k+\min (j, l)-1)$. 
(6) $\operatorname{IN}\left(\left\langle a^{i} b^{j}\right\rangle,\left\langle a^{k} B^{l}\right\rangle\right)=i l+k j$.

(7) $\operatorname{IN}\left(\left\langle a^{i} b^{j} a^{k} b^{l}\right\rangle,\left\langle a^{m} B^{n}\right\rangle\right)=(i+k) n+m(j+l)$.

\section{Corollary 3.7.}

$$
\operatorname{SI}\left(\left\langle a^{i} b^{j} a^{k} b^{l}\right\rangle\right) \begin{cases}=1 & \text { if } i=k \text { and } j=l \\ & \text { and } i=1 \text { or } j=1 \\ \leq(i+k-1)(j+l-1)-4 & \text { if } k=i \geq 2 \text { and } l=j \geq 2, \\ \leq(i+k-1)(j+l-1)-2 & \text { if } i \neq k \text { or } j \neq l .\end{cases}
$$

Proof. It follows from Proposition 3.6 (2) that if $i=k$ and $j=l$ and either pair is 1, then the $\mathrm{SI} \leq 1$; and if both are $\geq 2$ then

$$
\begin{aligned}
(i+k-2)(j+l-2)+1 & =(i+k-1)(j+l-1)-(i+k-1)-(j+l-1)+2 \\
& \leq(i+k-1)(j+l-1)-4 .
\end{aligned}
$$

If $i \neq k$ or $j \neq l$ then Proposition 3.6 (2) gives

$$
\begin{aligned}
\operatorname{SI}\left(\left\langle a^{i} b^{j} a^{k} b^{l}\right\rangle\right) & =(i+k-2)(j+l-2)+|i-k|+|j-l|-1 \\
& =(i+k-1)(j+l-1)-(i+k)+1-(j+l)+1+1+|i-k|+|j-l|-1 \\
& =(i+k-1)(j+l-1)-2 \min (i, k)-2 \min (j, l)+2 \\
& \leq(i+k-1)(j+l-1)-2 .
\end{aligned}
$$

The next remark is useful in the proof of Proposition A.6.

Remark 3.8. In the punctured torus, it follows from Definition 3.2 that if $\mathrm{P}=r$ susR, where $r$ and $s$ are distinct letters and $\mathrm{u}$ is an arbitrary linear word, then $\{\mathrm{P}, \mathrm{Q}\}$ is not a linked pair for any $\mathrm{Q}$.

\section{Proof of Theorem 1.10}

\subsection{Detailed Strategy of Proof}

This subsection amplifies the sketch presented in Subsection 1.2, continuing with the notation from Definition 1.9 and Section 2.

Given an arbitrary reduced cyclic word, we prove that its self-intersection number must be less than or equal to that of a word of the same length with few enough block-pairs to be amenable to a linked-pair self-intersection-number calculation. 
This "amalgamate and conquer" strategy is implemented by cross-corner surgery, which reduces the number of block-pairs in $w$ while conserving $\alpha(w)$ and $\beta(w)$ and increasing $\mathrm{SI}(w)$.

The detailed procedure at each step in the reduction depends on the number of different letters occurring in the word Figure 8). As we will see,

- a word that uses all four letters is always a candidate for cross-corner surgery using opposite corners with reversed orientation; the result will be a single word with one less block-pair (since this surgery reverses the orientation of part of the word, the number of different letters may change);

- if a word uses exactly three of the four letters and has at least five block-pairs, or if it uses only two of the four letters and has at least three block-pairs, then two cross-corner surgeries will reduce the number of block pairs by two (the intermediate stage is a two-component multi-word) and increase the self-intersection number by at least two; these surgeries permute the letters in the word, and so the new word still uses three letters or two letters if the old one did.

So the words remaining are:

- words with three letters and

(a) 4 block-pairs $(\langle$ rsrsrsrS $\rangle,\langle$ rsrsrSrS $\rangle$ and $\langle$ rsrSrsrS $\rangle)$,

(b) 3 block-pairs $(\langle$ rsrsrS $\rangle)$, or

(c) 2 block-pairs $(\langle\mathrm{rsrS}\rangle)$;

- words with two letters and

(d) 2 block-pairs $(\langle$ rsrs $\rangle)$ or

(e) 1 block-pair $(\langle r s\rangle)$.

- pure powers.

\subsection{Preparatory Lemmas}

In these lemmas and their proofs, Notation 3.1 will be used.

Lemma 4.1. If a reduced cyclic word $w$ contains all four letters $a, A, b, B$ then there exists a word $w^{\prime}$ with the same $\alpha$ and $\beta$ values, with one less block-pair, and with $\mathrm{SI}\left(w^{\prime}\right) \geq \mathrm{SI}(w)+1$. 


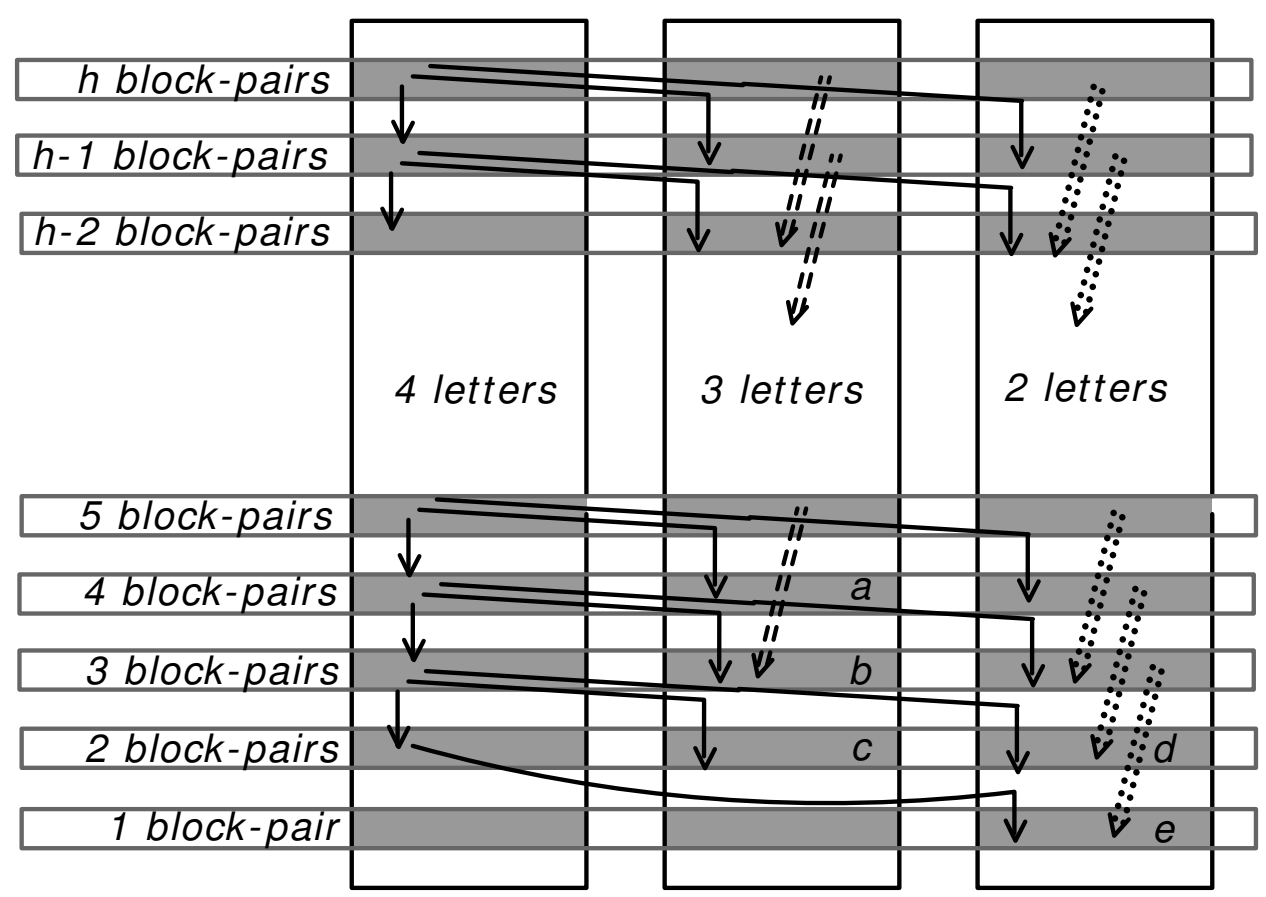

Figure 8: Flow chart of proof. The arrows correspond to possible cross-corner surgeries. Straight-line-arrows: Lemma 4.1; dashed arrows: Lemma 4.2; dotted arrows: Lemma 4.4. Terminal cases: $(\mathrm{a})=$ Lemma 4.3; (b) = Proposition 3.6 (5); (c) = Proposition 3.6 (3); $(\mathrm{d})=$ Corollary 3.7; (e) = Proposition 3.6 (1). Note that the self-intersection number of a pure power (word with 1 letter) can be calculated directly $\left(\mathrm{SI}\left(r^{k}\right)=k-1\right)$ and then compared with the maximum for general words of the same length; see Remark 1.4.

Proof. Claim: such a word must contain two corners with reverse orientation. In fact, let $w$ be a reduced cyclic word which contains all four letters (such a word must have at least two block-pairs) and which does not contain two subwords of the form $x y$ and $X Y$, where $x \in\{a, A\}$ and $y \in\{b, B\}$ or vice-versa. Now $w$ must contain at least one of $a b$ and $a B$; suppose $w$ contains $a b$. Then $w$ does not contain $A B$. So every B-block must be preceded by an $a$. Since there is at least one such block, $w$ must contain $a B$, which implies that $w$ does not contain $A b$. Since $w$ does not contain $A B$ or $A b$, there is no letter possible after an A-block. Since there is at least one such block, our hypothesis leads to a contradiction.

The lemma now follows from Proposition 2.2,(1).

Lemma 4.2. Suppose a cyclic word $w$ uses exactly three distinct letters from the set $\{a, A, b, B\}$ and has five or more block-pairs. Then there exists a word $w^{\prime}$, with two fewer block-pairs, with the same $\alpha$ and $\beta$ values, and such that $S I\left(w^{\prime}\right) \geq \operatorname{SI}(w)+2$.

Proof. Suppose the three letters are $a, b$ and $B$. The block-pairs are either ab's or aB's. 
We may suppose there are at least three ab's. Hence $w$ has the form $\langle a b a u a b$ av $a b$ ay where $\mathrm{u}, \mathrm{v}, \mathrm{y}$ represent (possibly empty) blocks of letters.

We pick two consecutive ab block-pairs and apply Proposition 2.2 (Cross Corner Surgery) as follows: $\langle\mathrm{a}| \mathrm{b}$ au $\mathrm{ab} \mid \mathrm{av}$ ab ay $\rangle \rightarrow[\langle\mathrm{b}$ au $\mathrm{ab} \mid\rangle,\langle\mathrm{a}| \mathrm{av}$ ab ay $\rangle]=[\langle$ baua $\rangle,\langle$ avabay $\rangle]$ $=\left[v^{\prime}, v^{\prime \prime}\right]$.

We have lost one $a b$ corner and one $b a$ corner, so the number of block-pairs has gone down by one. On the other hand, Proposition 2.2 guarantees that $\mathrm{SI}\left(\left[v^{\prime}, v^{\prime \prime}\right]\right) \geq$ $\mathrm{SI}(w)+1$.

Our consecutive corner condition guarantees that both $v^{\prime}$ and $v^{\prime \prime}$ contain both $a b$ and $b a$, so the multi-word $\left[v^{\prime}, v^{\prime \prime}\right]$ is a candidate for a second surgery, for example: $[\langle|$ baua $\rangle,\langle$ avab $|$ ay $\rangle] \rightarrow\langle|$ baua $\mid$ ayavab $\rangle=\langle$ bauayava $\rangle=w^{\prime}$.

We have lost another pair of corners, so the number of block-pairs has gone down by one more; Proposition 2.2 guarantees that $\mathrm{SI}\left(w^{\prime}\right) \geq \mathrm{SI}\left(\left[v^{\prime}, v^{\prime \prime}\right]\right)+1$, and thus $\mathrm{SI}\left(w^{\prime}\right) \geq$ $\mathrm{SI}(w)+2$. The $\alpha$ and $\beta$ values are clearly the same.

Lemma 4.3. If $w$ has one of the forms $\langle a b a b a b a B\rangle,\langle a b a B a b a B\rangle,\langle a b a b a B a B\rangle$ then there exists a word $w^{\prime}$ with two block-pairs, the same $\alpha$ and $\beta$ as $w$ and such that $\mathrm{SI}\left(w^{\prime}\right) \geq \mathrm{SI}(w)+2$.

Proof. 1. $\langle$ abababaB $\rangle$. We apply cross corner surgery (Proposition 2.2) as follows: $\langle\mathrm{ab}|\mathrm{aba}| \mathrm{baB}\rangle \rightarrow[\langle\mathrm{ab} \mid \mathrm{baB}\rangle,\langle| \mathrm{aba}]=\left[\langle\mathrm{abaB}\rangle,\langle\mathrm{ba}]=\left[v^{\prime}, v^{\prime \prime}\right]\right.$. The multiword $\left[v^{\prime}, v^{\prime \prime}\right]$ has the same $\alpha$ and $\beta$ values as $w$. Furthermore, $\operatorname{SI}\left(\left[v^{\prime}, v^{\prime \prime}\right]\right) \geq \operatorname{SI}(w)+1$. Another application of Proposition 2.2: $[\langle\mathrm{a} \mid \mathrm{baB}\rangle,\langle\mathrm{b} \mid \mathrm{a}\rangle] \rightarrow\langle\mathrm{a}|\mathrm{ab}| \mathrm{baB}\rangle=\langle\mathrm{abaB}\rangle=w^{\prime}$ yields a word $w^{\prime}$ with two block-pairs, the same $\alpha$ and $\beta$ values as $w$, and $\operatorname{SI}\left(w^{\prime}\right) \geq$ $\mathrm{SI}(w)+2$.

2. $\langle$ abaBabaB $\rangle$. Cross-corner surgery $\langle a b a B|a b a| B\rangle \rightarrow[\langle a b a B \mid B\rangle,\langle a b a \mid\rangle]=[\langle a b a B\rangle,\langle b a\rangle]$ leads to the same half-way step as the previous case.

3. $\langle$ ababaBaB $\rangle$. Apply Proposition 2.2,

$$
\langle\text { ababa }|\mathrm{BaB}|\rangle \rightarrow[\langle\text { ababa } \mid\rangle,\langle\mathrm{BaB} \mid\rangle]=[\langle\mathrm{abab}\rangle,\langle\mathrm{aB}\rangle]=\left[\left\langle a^{i} b^{j} a^{k} b^{l}\right\rangle,\left\langle a^{m} B^{n}\right\rangle\right],
$$

say, $($ so $\alpha(w)=i+k+m, \beta(w)=j+l+n)$, and

$$
\mathrm{SI}(w) \leq \mathrm{SI}\left(\left[\left\langle a^{i} b^{j} a^{k} b^{l}\right\rangle,\left\langle a^{m} B^{n}\right\rangle\right]\right)-1 \text {. }
$$

Now

$$
\mathrm{SI}\left(\left[\left\langle a^{i} b^{j} a^{k} b^{l}\right\rangle,\left\langle a^{m} B^{n}\right\rangle\right]\right)=\mathrm{SI}\left(\left\langle a^{i} b^{j} a^{k} b^{l}\right\rangle\right)+\mathrm{SI}\left(\left\langle a^{m} B^{n}\right\rangle\right)+\operatorname{IN}\left(\left\langle a^{i} b^{j} a^{k} b^{l}\right\rangle,\left\langle a^{m} B^{n}\right\rangle\right) .
$$

Because of the format of Corollary 3.7 we need to consider two cases: 
(i) $i=k$ and $j=l=1$ (by the construction, $i$ and $k$ cannot be 1 ). In that case $\operatorname{SI}\left(\left\langle a^{i} b^{j} a^{k} b^{l}\right\rangle\right)=1$. By Proposition 3.6 (7), $\operatorname{IN}\left(\left\langle a^{i} b^{j} a^{k} b^{l}\right\rangle,\left\langle a^{m} B^{n}\right\rangle\right)=$ $(i+k) n+m(j+l)$, and by Proposition 3.6 (1) $\operatorname{SI}\left(\left\langle a^{m} B^{n}\right\rangle\right)=(m-1)(n-1)$. This gives

$\mathrm{SI}\left(\left[\left\langle a^{i} b a^{i} b\right\rangle,\left\langle a^{m} B^{n}\right\rangle\right]\right)=1+2 i n+2 m+(m-1)(n-1)=(2 i-1) n+m(n+1)+2$

and $\operatorname{SI}(w) \leq(2 i-1) n+m(n+1)+1$. On the other hand the word $w^{\prime}=\left\langle a^{2 i} b^{2} a^{m} B^{n}\right\rangle$ has the same $\alpha$ and $\beta$-values as $w$ and (Proposition 3.6 (3)) $\mathrm{SI}\left(w^{\prime}\right)=(2 i+m-1)(n+1)=(2 i-1) n+m(n+1)+(2 i-1)$. Since as remarked above $i \geq 2$, it follows that $\mathrm{SI}\left(w^{\prime}\right) \geq \mathrm{SI}(w)+2$.

(ii) For all other $\left\langle a^{i} b^{j} a^{k} b^{l}\right\rangle$, Corollary 3.7 gives $\operatorname{SI}\left(\left\langle a^{i} b^{j} a^{k} b^{l}\right\rangle\right) \leq(i+k-1)(j+$ $l-1)-2$, and

$$
\begin{gathered}
\mathrm{SI}\left(\left[\left\langle a^{i} b a^{i} b\right\rangle,\left\langle a^{m} B^{n}\right\rangle\right]\right) \leq(i+k-1)(j+l-1)-2+(i+k) n+m(j+l)+(m-1)(n-1) \\
=(i+k+m-1)(j+l+n-1)-1,
\end{gathered}
$$

so $\mathrm{SI}(w) \leq(i+k+m-1)(j+l+n-1)-2$. Comparing this estimate with $\mathrm{SI}\left(\left\langle a^{i+k} b^{j+l} a^{m} B^{n}\right\rangle\right)=(i+k+m-1)(j+l+n-1)$ (Proposition 3.6 (3) again) completes the proof.

Lemma 4.4. If $w$ uses exactly two letters and $w$ has three or more block-pairs, then there exists a word $w^{\prime}$ with two fewer block-pairs, with the same $\alpha$ and $\beta$ values, and such that $\mathrm{SI}\left(w^{\prime}\right) \geq \mathrm{SI}(w)+2$.

Proof. Suppose the two letters are $a$ and $b$, so $w=\langle$ ababab... $\rangle$. Now proceed as in the proof of Lemma 4.2 .

\subsection{End of the proof}

Proposition 4.5. Let $w$ be the reduced cyclic word corresponding to a free homotopy class of curves on the punctured torus, with $h(w)=h>0$. Then there exists a word $w^{\prime}$ such that $w^{\prime}$ has one or two blocks, $\alpha\left(w^{\prime}\right)=\alpha(w)$ and $\beta\left(w^{\prime}\right)=\beta(w)$, and $\mathrm{SI}\left(w^{\prime}\right) \geq \mathrm{SI}(w)+h-2$.

Proof. If $h=1$ or 2 , then taking $w^{\prime}=w$ satisfies the conclusions of the Proposition.

For $h>2$, we proceed by complete induction, and assume that the result holds for any word with a number of block-pairs smaller than $h$. Since $h$ is positive, $w$ contains 2, 3 or 4 distinct letters. We consider the cases separately. 
(2 letters) Suppose that $w$ contains exactly two distinct letters. If $w$ has more than two block-pairs, then by Lemma 4.4 there exists a word $v$ with $h-2$ block-pairs, with the same $\alpha$ and $\beta$ values, and such that $\operatorname{SI}(v) \geq \operatorname{SI}(w)+2$. By the induction hypothesis, there exists $w^{\prime}$ with one or two blocks, the same $\alpha$ and $\beta$ as $v$ and such that $\mathrm{SI}\left(w^{\prime}\right) \geq \operatorname{SI}(v)+(h-2)-2 \geq \operatorname{SI}(w)+h-2$, as desired.

(3 letters) Suppose that $w$ contains exactly three distinct letters. If $h>4$, the result follows from combining Lemma 4.2 and the induction hypothesis. If $h=4$ then $w$ must have one of the following forms: $\langle a b a b a b a B\rangle,\langle a b a B a b a B\rangle$ or $\langle a b a b a B a B\rangle$. Lemma 4.3 covers these three cases. In the case $h=3$, the word can be supposed to be $w=\left\langle a^{i} b^{j} a^{k} b^{l} a^{m} B^{n}\right\rangle$. Taking $w^{\prime}=\left\langle a^{i+k} b^{j+l} a^{m} B^{n}\right\rangle$, and applying Proposition $3.6(3)$ and (5), yields the desired result.

(4 letters) Suppose that $w$ contains all four letters $a, b, A, B$. By Lemma 4.1, there exists a word $v$ with $h-1$ block-pairs, with $\alpha(v)=\alpha(w), \beta(v)=\beta(w)$ and such that $\operatorname{SI}(v) \geq \operatorname{SI}(w)+1$. Now the result follows from our induction hypothesis. More explicitly, there exists a word $w^{\prime}$, with same $\alpha$ and $\beta$ as $v$, with one or two block-pairs and such that $\mathrm{SI}\left(w^{\prime}\right) \geq \mathrm{SI}(v)+(h-1)-2 \geq \operatorname{SI}(w)+h-2$.

Proof of Theorem 1.10. If $h=1$ or 2 the result follows from Proposition 3.6 (1-4). So suppose that $h>2$, By Proposition 4.5, there exists a word $w^{\prime}$ with $\alpha\left(w^{\prime}\right)=\alpha(w)$ and $\beta\left(w^{\prime}\right)=\beta(w), \mathrm{SI}(w) \leq \mathrm{SI}\left(w^{\prime}\right)-h+2<\mathrm{SI}\left(w^{\prime}\right)$, and such that $w^{\prime}$ has one or two blocks. Referring to Proposition 3.6(1-4), any such word satisfies $\mathrm{SI}\left(w^{\prime}\right) \leq$ $\left(\alpha\left(w^{\prime}\right)-1\right)\left(\beta\left(w^{\prime}\right)-1\right)$. This proves part (1).

Part (2) of the theorem follows also, by inspection, from Proposition 3.6(1-4).

\subsection{Words with sub-maximal intersection number}

Lemma 4.6. If $w$ is one of the following words: $\langle a b a b A B\rangle,\langle a b A b a B\rangle,\langle a b a B A B\rangle$, and $\langle\mathrm{abABaB}\rangle$, then

$$
\mathrm{SI}(w) \leq(\alpha(w)-1)(\beta(w)-1)-2
$$

Proof. Proposition 3.6 and Corollary 3.7 can be applied after one or two cross-corner surgeries (Proposition 2.2), each of which increases the self-intersection number by at least one:

- $\left\langle a^{i} b^{j} a^{k} b^{l}\left|A^{m} B^{n}\right|\right\rangle \rightarrow\left\langle a^{i} b^{j} a^{k} b^{l}\left|b^{n} a^{m}\right|\right\rangle=\left\langle a^{i+m} b^{j} a^{k} b^{l+n}\right\rangle$

- $\left\langle a^{i} b^{j}\left|A^{k} b^{l} a^{m} B^{n}\right|\right\rangle \rightarrow\left\langle a^{i} b^{j}\left|b^{n} A^{m} B^{l} a^{k}\right|\right\rangle=\left\langle a^{i+k} b^{n+j} A^{m} B^{l}\right\rangle$ 
- $\left\langle a^{i} b^{j} a^{k}\left|B^{l} A^{m} B^{n}\right|\right\rangle \rightarrow\left[\left\langle a^{i} b^{j} a^{k} \mid\right\rangle,\left\langle B^{l} A^{m} B^{n} \mid\right\rangle\right]=\left[\left\langle a^{i+k} b^{j}\right\rangle,\left\langle A^{m} B^{n+l}\right\rangle\right]$ $\left[\left\langle a^{i+k} \mid b^{j}\right\rangle,\left\langle A^{m} \mid B^{n+l}\right\rangle\right] \rightarrow\left\langle a^{i+k}\left|a^{m} b^{n+l}\right| b^{j}\right\rangle=\left\langle a^{i+k+m} b^{j+n+l}\right\rangle$.

- $\left\langle a^{i}\left|b^{j} A^{k}\right| B^{l} a^{m} B^{n}\right\rangle \rightarrow\left\langle a^{i}\left|a^{k} B^{j}\right| B^{l} a^{m} B^{n}\right\rangle=\left\langle a^{i+k} B^{l+j} a^{m} B^{n}\right\rangle$

The following lemma will be used in the proof of Theorem 4.9. Note that the special case it covers admits a bound for the self-intersection number sharper than that of Theorem 1.11.

Lemma 4.7. If $w$ is a word with three block-pairs, then $\operatorname{SI}(w) \leq(\alpha(w)-1)(\beta(w)-$ $1)-2$. In particular, if length $L=\alpha(w)+\beta(w)$ then

$$
\mathrm{SI}(w) \leq \begin{cases}(L-2)^{2} / 4-2 & \text { if } L \text { is even } \\ (L-1)(L-3) / 4-2 & \text { if } L \text { is odd }\end{cases}
$$

Proof. Without loss of generality, we may suppose that the number $N$ of $\mathrm{A}$ and $\mathrm{B}$ blocks in $w$ is at most three.

If $N=0$, the result follows from Lemma 4.4 and Theorem 1.11 .

If $N=1$, we may suppose that $w=\langle$ ababaB $\rangle$ which is covered by Proposition 3.6(5).

If $N=2$, we may suppose that $w$ is one of $\langle\mathrm{abAbaB}\rangle$ or $\langle\mathrm{ababAB}\rangle$; if $N=3$, we may suppose that $w$ is one of $\langle a b a B A B\rangle$ or $\langle a b A B a B\rangle$; for these cases, the result follows from Lemma 4.6.

Lemma 4.8. Let $w$ be a word with two block-pairs and two letters, say a and $b$ with length $L \geq 4$. Either $L=4$ and $w=a b a b$ with $\mathrm{SI}(w)=1$, or

$$
\mathrm{SI}(w) \leq \begin{cases}(L-2)^{2} / 4-2 & \text { if } L \text { is even } \\ (L-1)(L-3) / 4-2 & \text { if } L \text { is odd }\end{cases}
$$

Proof. Refer to Corollary 3.7 ,

First notice that $a b^{j} a b^{j}$ has length $L=2 j+2$, an even number, and if $j \geq 2$

$$
(L-2)^{2} / 4-2=j^{2}-2 \geq 1=\operatorname{SI}\left(a b^{j} a b^{j}\right) .
$$

So the lemma holds for all words of the form $a b^{j} a b^{j}$ and $a^{i} b a^{i} b$.

For the rest of the words in question, $\operatorname{SI}(w) \leq(i+k-1)(j+l-1)-2$, so the result follows as in Remark 1.13.

Theorem 4.9. Let $w$ be a primitive reduced cyclic word of length $L>3$ and selfintersection number

$$
\mathrm{SI}(w)= \begin{cases}(L-2)^{2} / 4-1 & \text { if } L \text { is even } \\ (L-1)(L-3) / 4-1 & \text { if } L \text { is odd }\end{cases}
$$


i.e. one less than the maximum possible for its length. Then if $L$ is odd, $w=r^{i} s^{j} R^{k} S^{l}$ with $i+k=\frac{L-1}{2}$ or $\frac{L+1}{2}$.

And if $L$ is even, $w$ has one of the following forms.

(1) $\left\langle r^{L / 2-1} s^{L / 2+1}\right\rangle$;

(2) $\left\langle r^{i} s^{j} R^{k} S^{l}\right\rangle, i+k=\frac{L}{2}$;

(3) $\left\langle r^{i} s^{j} r^{k} S^{l}\right\rangle, i+k=\frac{L}{2}-1$ or $\frac{L}{2}+1$.

Here $r=a$ or $A$ and $s=b$ or $B$, or vice-versa.

Remark 4.10. The primitive reduced cyclic words of length $L \leq 3$, namely those of the form $a, a b, a b b$ all have self-intersection number zero, the maximum for those lengths (cf. Table 1).

Proof of Theorem 4.9 By Proposition 4.5 and Lemma 4.7, $h(w)=1$ or 2 (the only primitive words with zero block-pairs are singletons, which do not satisfy the hypothesis.).

We begin with the case $h(w)=2$. By Lemma 4.8, we can assume that $w=$ $\langle a b a B\rangle$ or $\langle a b A B\rangle$.

First suppose $w=\langle\mathrm{abaB}\rangle$. By Proposition 3.6 (3), $\operatorname{SI}(w)=(\alpha(w)-1)(\beta(w)-1)$.

If $L$ is odd, then $\operatorname{SI}(w)=(L-1)(L-3) / 4-1$. Since $\beta(w)=L-\alpha(w)$, it follows that $(L-1)(L-3) / 4-1=(\alpha(w)-1)(L-\alpha(w)-1)$. This implies $\alpha(w)=(L \pm \sqrt{5}) / 2$, which is not an integer, a contradiction.

So $L$ is even, and $(L / 2-1)^{2}-1=(\alpha(w)-1)(L-\alpha(w)-1)$. This implies $\alpha(w)=\frac{n}{2}-1$ or $\frac{n}{2}+1$, as desired.

Now suppose $w=\langle\mathrm{abAB}\rangle$. The result follows from Proposition 3.6 (4). This settles the case $h(w)=2$.

If $h(w)=1$ then by Proposition 3.6(1), $\operatorname{SI}(w)=(\alpha(w)-1)(L-\alpha(w)-1)$. The solutions of the equation

$$
(\alpha(w)-1)(L-\alpha(w)-1)=(L-1)(L-3) / 4-1
$$

are $\alpha(w)=\frac{L-\sqrt{5}}{2}$ and $\alpha(w)=\frac{L+\sqrt{5}}{2}$. Hence there are no words of sub-maximal selfintersection with odd length $L$ and one block-pair.

On the other hand, the solutions of the equation

$$
(\alpha(w)-1)(L-\alpha(w)-1)=(L / 2-1)^{2}-1
$$

are $\alpha(w)=L / 2-1$ and $L / 2+1$; the result follows. 
Theorem 4.11. If $L$ is odd, there are $(L-1)(L-3)$ distinct reduced cyclic words with self-intersection number one less than the maximum for their length.

If $L$ is even, there are $5(L-2)^{2} / 2$ distinct reduced cyclic words with self-intersection number one less than the maximum for their length.

Proof. Refer to Theorem 4.9. Suppose $L$ is odd. If $i+k=\frac{L-1}{2}$, there are $1, \ldots, \frac{L-3}{2}$ possibilities for $i$, and $1 \ldots, \frac{L-1}{2}$ possibilities for $j$. The total is $\frac{L-3}{2} \frac{L-1}{2}$. Interchanging the roles of $i$ and $j$, and those of $a$ and $b$, we obtain $(L-1)(L-3)$.

Suppose $L$ is even: there are 8 words of the form $\left\langle r^{L / 2-1} s^{L / 2+1}\right\rangle$, together with $2(L / 2-1)^{2}$ words of the form $\left\langle r^{i} s^{j} R^{k} S^{l}\right\rangle$ and $4 L(L / 2-2)$ words of the form $\left\langle r^{i} s^{j} r^{k} S^{l}\right\rangle$; the total is $5 L^{2} / 2-10 L+10=5(L-2)^{2} / 2$.

Remark 4.12. The leading coefficient of the polynomial expression for the number of maximal words of odd length is two times larger than that for even length, whereas for sub-maximal words the even leading coefficient is 2.5 times the odd leading coefficient. The discrepancies balance out to some extent, when one considers maximal and submaximal words together. For odd length $L$, this number is $3 L^{2}-12 L+17$, while for even length it is $7 L^{2} / 2-14 L+18$.

\section{Computational results and conjectures for the pair of pants}

The "pair of pants" is the usual name for the surface with boundary, homeomorphic to the thrice-punctured sphere. The same computational methods that yielded Computational Theorem 1.7 suggest that the dependance of maximum self-intersection number on length for the pair of pants is quadratic, just as it was for the punctured torus.

Computational Theorem 5.1. For lengths $L \leq 15$, the maximal self-intersection number of primitive reduced cyclic words of length $L$ on the pair of pants is:

$$
\begin{cases}\left(L^{2}-1\right) / 4 & \text { if } L \text { is odd } \\ L^{2} / 4-1 & \text { if } L \equiv 0 \quad(\bmod 4) \\ L^{2} / 4-2 & \text { if } L>2 \text { and } L \equiv 2 \quad(\bmod 4) \\ 1 & \text { if } L=2 .\end{cases}
$$

Moreover, if $L$ is odd, the words realizing the maximal self-intersection number are $r(r s)^{\frac{L-1}{2}}$, where $\{r, s\}=\{a, B\}$ or $\{r, s\}=\{A, b\}$. (primitive words of even length follow a more complicated pattern, which cannot be easily reduced to a formula).

Removing the restriction "primitive" leads to: 
Computational Theorem 5.2. For $L \leq 15$ the maximal self-intersection number for a reduced cyclic word of length $L$ on the pair of pants is:

$$
\begin{cases}\left(L^{2}-1\right) / 4 & \text { if } L \text { is odd } \\ L^{2} / 4+L / 2-1 & \text { if } L \text { is even }\end{cases}
$$

Moreover

(1) If $L$ is odd, the words realizing the maximal self-intersection number are $r(r s)^{\frac{L-1}{2}}$, where $\{r, s\}=\{a, B\}$ or $\{r, s\}=\{A, b\}$

(2) If $L$ is even, the words realizing the maximal self-intersection number are $(A b)^{\frac{L-1}{2}}$ and $(a B)^{\frac{L-1}{2}}$.

The next two computational theorems show radically different behavior from what we know for the punctured torus.

Computational Theorem 5.3. The number of distinct free homotopy classes of curves on the pair of pants of length $L$ realizing the maximal self-intersection number is

$$
\begin{cases}2 & \text { if } L \text { is even, } \\ 4 & \text { if } L \text { is odd. }\end{cases}
$$

Computational Theorem 5.4. For $L \leq 15$ the minimal self-intersection number for the free homotopy class on the pair of pants representing a primitive reduced cyclic word of length $L$ is 0 for $L=1,2$ and $[L / 2]$ (the integer part of $L / 2$ ) for $L \geq 3$.

It is reasonable to conjecture that all this behavior will persist for higher values of $L$.

Remark 5.5. Note that an analogue of Proposition 2.2 can be proved for any surface with boundary. So words with maximal self-interesection number cannot contain (the generalization of) diagonally opposed corners with reversed orientations.

\section{A Appendix: Proof of Proposition 3.6}

The seven parts of Proposition 3.6 are proved separately as Propositions A.1, A.2, A.3, A.4, A.5, A.6, A.7. The method of proof for each of these propositions is via Theorem 3.4; a counting of all occurrences of each of the three types of linked pairs given in Definition 3.2 .

I. These pairs are easy to count. They have the form $\{r r, s s\}$, where $r \in\{a, A\}$ and $s \in\{b, B\}$. 
II. These have the form $\left\{p_{1} \mathrm{y} p_{2}, q_{1} \mathrm{y} q_{2}\right\}$, with $p_{1} \neq p_{2}$ and $q_{1} \neq q_{2}$. One locates all subwords y with two occurrences and ckecks for each pair if the corresponding $p_{1} \mathrm{y} p_{2}$ and $q_{1} \mathrm{y} q_{2}$ are linked.

III. Analogously, these pairs are found by locating subwords y which occur in our word or multiword along with their inverse $\mathrm{Y}$. Such a pair will contribute to the count if the corresponding $p_{1} y p_{2}$ and $\bar{q}_{2} y \bar{q}_{1}$ are linked, see Remark 3.3.

Proposition A.1. $\operatorname{SI}\left(\left\langle a^{i} b^{j}\right\rangle\right)=(i-1)(j-1)$.

Proof. There are $i-1$ occurrences of $a a$ and $j-1$ occurrences of $b b$ in $\left\langle a^{i} b^{j}\right\rangle$. Thus there are $(i-1)(j-1)$ pairs of type I. There are no pairs of the other two types.

Proposition A.2. $\operatorname{SI}\left(\left\langle a^{i} b^{j} a^{k} b^{l}\right\rangle\right)=(i+k-2)(j+l-2)+1$ if $k=i$ and $j=l$; and $(i+k-2)(j+l-2)+|i-k|+|j-l|-1$, otherwise.

Proof. I. There are $(i+k-2)(j+l-2)$ pairs of this kind.

II. In this case, $\mathrm{y} \in\left\{a^{K}, B^{K}, a^{K} b^{J}, b^{K} a^{J}, a^{K} b^{J} a^{L}, b^{J} a^{K} b^{L}\right\}$ for some positive integers $J, K$ and $L$.

(i) $\mathrm{y}=a^{K}$. Analysis: Table 5, using Table 2, The total number is $|i-k|-1$ if $i \neq k$ and zero otherwise.

\begin{tabular}{|c|c|c|c|}
\hline configuration & with & if & add \\
\hline$\left\{a^{k+2}, b a^{k} b\right\}$ & $a^{k+2}$ in $a^{i}$ & $k+2 \leq i$ & $i-k-1$ \\
\hline$\left\{a^{i+2}, b a^{i} b\right\}$ & $a^{i+2}$ in $a^{k}$ & $i+2 \leq k$ & $k-i-1$ \\
\hline
\end{tabular}

Table 5: Linked pairs in $\left\langle a^{i} b^{j} a^{k} b^{l}\right\rangle$ of type II with $\mathrm{y}=a^{K}$.

(ii) $\mathrm{y}=b^{K}$. With similar arguments as in the case (i), it can be shown that the number of pairs here is $|j-l|-1$ if $j \neq l$ and zero otherwise.

(iii) $\mathrm{y}=a^{K} b^{J}$. By Table 3, the linked words of pairs with this y have the form $b a^{K} b^{J} b$ and $a a^{K} b^{J} a$. Analysis: Table 6 .

\begin{tabular}{|c|c|c|c|}
\hline configuration & with & if & add \\
\hline$\left\{b a^{i} b^{l} b, a a^{i} b^{l} a\right\}$ & $b a^{i} b^{l} b$ in $b a^{i} b^{j}, a a^{i} b^{l} a$ in $a^{k} b^{l} a$ & $i<k$ and $j>l$ & 1 \\
\hline$\left\{b a^{k} b^{j} b, a a^{k} b^{j} a\right\}$ & $b a^{k} b^{j} b$ in $b a^{k} b^{l}, a a^{k} b^{j} a$ in $a^{i} b^{j} a$ & $k<i$ and $j<l$ & 1 \\
\hline
\end{tabular}

Table 6: Linked pairs in $\left\langle a^{i} b^{j} a^{k} b^{l}\right\rangle$ of type II with $\mathrm{y}=a^{K} b^{J}$. The three types of linked pairs can be added as follows:

(iv) $\mathrm{y}=b^{K} a^{J}$. By Table 3, the linked words have the form $a b^{K} a^{J} a$ and $b b^{K} a^{J} b$. Analysis: Table 7 .

By (iii) and (iv) we add 1 if $k \neq i$ and $j \neq l$. 


\begin{tabular}{|c|c|c|c|}
\hline configuration & with & if & add \\
\hline$\left\{b b^{j} a^{i} b, a b^{j} a^{i} a\right\}$ & $b b^{j} a^{i} b$ in $b^{l} a^{i} b, a b^{j} a^{i} a$ in $a b^{j} a^{k}$ & $i<k$ and $j<l$ & 1 \\
\hline$\left\{b b^{l} a^{k} b, a b^{l} a^{k} a\right\}$ & $b b^{l} a^{k} b$ in $b^{j} a^{k} b, a b^{l} a^{k} a$ in $a b^{l} a^{i}$ & $k<i$ and $j>l$ & 1 \\
\hline
\end{tabular}

Table 7: Linked pairs in $\left\langle a^{i} b^{j} a^{k} b^{l}\right\rangle$ of type II with $\mathrm{y}=b^{K} a^{J}$.

(v) y $=a^{K} b^{J} a^{L}$. Since y has two occurrences, $j=l$. By Table 4 the linked pairs have the form $\left\{a a^{K} b^{j} a^{L} a, b a^{K} b^{j} a^{L} b\right\}$. There are two pairs of this kind, namely $\left\{a a^{i} b^{j} a^{k} a, b a^{i} b^{j} a^{k} b\right\}$ and $\left\{a a^{k} b^{l} a^{i} a, b a^{k} b^{l} a^{i} b\right\}$. Each of the possibilities implies that $i<k$ and $k<i$. Hence, such pairs are not possible.

(vi) $\mathrm{y}=b^{K} a^{J} b^{L}$. As in case (v), there are no linked pairs of this form.

III. There are no pairs of type III because the word contains no occurrence of a letter and its inverse.

If $i=k$ and $j=l$ add 1 , because the word has the form $w^{2}$, where $w$ is a primitive word. Adding up all the contributions completes the proof.

Proposition A.3. IN $\left(\left\langle a^{i} b^{j}\right\rangle,\left\langle a^{m} B^{n}\right\rangle\right)=i n+m j$.

Proof. $\quad$ I. There are $(i-1)(n-1)+(j-1)(m-1)$ linked pairs of this type.

II. $\mathrm{y}=a^{K}$ for some positive integer $K$. Analysis: Table 8, using Table 2, The contributions of the different rows may be grouped in the following way: $(\mathrm{a}+\mathrm{c}$ $+\mathrm{d})=i-1,(\mathrm{~b}+\mathrm{f}+\mathrm{h})=m-1$ and $(\mathrm{e}+\mathrm{g}+\mathrm{i})=1$.

\begin{tabular}{|c|c|c|c|c|}
\hline & configuration & with & if & add \\
\hline a & $\left\{a^{K} b, B a^{K}\right\}$ & $a^{K} b$ in $a^{i} b, B a^{K}$ in $B a^{m}$ & $K \in\{2 \ldots \min (m, i)\}$ & $\min (m, i)-1$ \\
\hline $\mathrm{b}$ & $\left\{a^{i} B, b a^{i}\right\}$ & $b a^{K}$ in $b a^{i}, a^{K} B$ in $a^{m} B$ & $K \in\{2 \ldots \min (m, i)\}$ & $\min (m, i)-1$ \\
\hline $\mathrm{c}$ & $\left\{a^{m+2}, B a^{m} B\right\}$ & $a^{m+2}$ in $a^{i}$ & $m+2 \leq i$ & $i-m-1$ \\
\hline $\mathrm{d}$ & $\left\{a^{m+1} b, B a^{m} B\right\}$ & $a^{m+1}$ in $a^{i}$ & $m+1 \leq i$ & 1 \\
\hline $\mathrm{e}$ & $\left\{b a^{m+1}, B a^{m} B\right\}$ & $a^{m+1}$ in $a^{i}$ & $m<i$ & 1 \\
\hline $\mathrm{f}$ & $\left\{a^{i+1} B, b a^{i} b\right\}$ & $a^{i+1}$ in $a^{m}$ & $i<m$ & 1 \\
\hline $\mathrm{g}$ & $\left\{B a^{i+1}, b a^{i} b\right\}$ & $a^{i+1}$ in $a^{m}$ & $i+2 \leq m$ & $m-i-1$ \\
\hline $\mathrm{h}$ & $\left\{a^{i+2}, b a^{i} b\right\}$ & $a^{i+2}$ in $a^{m}$ & $i=m$ & 1 \\
\hline $\mathrm{i}$ & $\left\{b a^{i} b, B a^{m} B\right\}$ & & & \\
\hline
\end{tabular}

Table 8: Linked pairs of $\left\langle a^{i} b^{j}\right\rangle$ and $\left\langle a^{m} B^{n}\right\rangle$ of type II with $\mathrm{y}=a^{K}$.

III. $\mathrm{y}=b^{K}$. Combining Remark 3.3 with Table 2 we analyze these pairs in Table 9 . Here $(\mathrm{a}+\mathrm{c}+\mathrm{f})=n-1,(\mathrm{~b}+\mathrm{g}+\mathrm{i})=j-1$ and $(\mathrm{d}+\mathrm{e}+\mathrm{h})=1$.

Adding the contributions from each of the three types yields the result. 


\begin{tabular}{|c|c|c|c|c|}
\hline & configuration & with & if & add \\
\hline $\mathrm{a}$ & $\left\{a b^{K}, a B^{K}\right\}$ & $a b^{K}$ in $a b^{j}, a B^{K}$ in $a B^{n}$ & $K \in\{2 \ldots \min (j, n)\}$ & $\min (j, n)-1$ \\
\hline $\mathrm{b}$ & $\left\{b^{K} a, B^{K} a\right\}$ & $a b^{K}$ in $a b^{j}, a B^{K}$ in $a B^{n}$ & $K \in\{2 \ldots \min (j, n)\}$ & $\min (j, n)-1$ \\
\hline $\mathrm{c}$ & $\left\{a b^{j} a, B^{j+1} a\right\}$ & $B^{j+1} a$ in $B^{n} a$ & $j<n$ & 1 \\
\hline $\mathrm{d}$ & $\left\{a b^{j} a, a B^{j+1}\right\}$ & $a B^{j+1}$ in $a B^{n}$ & $j<n$ & 1 \\
\hline $\mathrm{e}$ & $\left\{a b^{j} a, a B^{n} a\right\}$ & & $j=n$ & 1 \\
\hline $\mathrm{f}$ & $\left\{a b^{j} a, B^{j+2}\right\}$ & $B^{j+2}$ in $B^{n}$ & $j+2 \leq n$ & $n-j-1$ \\
\hline $\mathrm{g}$ & $\left\{b^{n+1} a, a B^{n} a\right\}$ & $b^{n+1} a$ in $b^{j} a$ & $n<j$ & 1 \\
\hline $\mathrm{h}$ & $\left\{a b^{n+1}, a B^{n} a\right\}$ & $a b^{n+1}$ in $a b^{j}$ & $n<j$ & 1 \\
\hline $\mathrm{i}$ & $\left\{b^{n+2}, a B^{n} a\right\}$ & $b^{n+2}$ in $b^{j}$ & $n+2 \leq j$ & $j-n-1$ \\
\hline
\end{tabular}

Table 9: Linked pairs of $\left\langle a^{i} b^{j}\right\rangle$ and $\left\langle a^{m} B^{n}\right\rangle$ of type III with $\mathrm{y}=b^{K}$.

Proposition A.4. $\operatorname{SI}\left(\left\langle a^{i} b^{j} a^{k} B^{l}\right\rangle\right)=(i+k-1)(j+l-1)$.

Proof. I. There are $(i+k-2)(j-1)$ ocurrences of pairs $\{a a, b b\}$ and $(i+k-2)(l-1)$ ocurrences of pairs $\{a a, B B\}$. The total is $(i+k-2)(j+l-1)$.

II. y $\in\left\{a^{I}, b^{J}, B^{K}\right\}$ for some positive integers $I, J$ and $K$. There are no linked pairs with $\mathrm{y} \in\left\{a^{I}, b^{J}, B^{K}\right\}$. By Table 2, (interchanging roles of $a$ 's and $b$ 's) there are no pairs such that $\mathrm{y} \in\left\{b^{J}, B^{K}\right\}$. We analyze each of the possible pairs with $\mathrm{y}=a^{I}$ in Table 10, using Table 2, We show that there are $i+k-2$ of this type.

\begin{tabular}{|c|c|c|c|c|}
\hline & configuration & with & if & add \\
\hline & $\left\{a^{I} b, B a^{I}\right\}$ & $a^{I} b$ in $a^{i} b, B a^{I}$ in $B a^{i}$ & $I \in\{2,3, \ldots, i\}$ & $i-1$ \\
\hline & $\left\{b a^{I}, a^{I} B\right\}$ & $b a^{I}$ in $b a^{i}, a^{I} B$ in $a^{i} B$ & $I \in\{2,3, \ldots, k\}$ & $k-1$ \\
\hline
\end{tabular}

Table 10: Linked pairs of $\left\langle a^{i} b^{j} a^{k} B^{l}\right\rangle$ of type II with $\mathrm{y}=a^{I}$.

III. $\mathrm{y}=b^{J}$. Analysis: Table 11. The total contribution from the Type III linked pairs comes to $j+l-1$.

It follows that the total self-intersection number of $\left\langle a^{i} b^{j} a^{k} B^{l}\right\rangle$ is $(i+k-2)(j+l-$ $2)+i+k-2+j+l-1=(i+k-1)(j+l-1)$.

Proposition A.5. SI $\left(\left\langle a^{i} b^{j} A^{k} B^{l}\right\rangle=(i+k-1)(j+l-1)-1\right.$.

Proof. $\quad$ I. There are in total $(i+k-2)(j+l-2)$ pairs of this type.

II. In this case $\mathrm{y} \in\left\{a^{I}, b^{J}, B^{K}, A^{L}\right\}$ for some integers $I, J, K$ and $L$. Analysis: Table 12, using Table 2 . 


\begin{tabular}{|c|c|c|c|c|}
\hline & configuration & with & if & add \\
\hline & $\left\{b^{J} a, B^{J} a\right\}$ & $b^{J} a$ in $b^{j} a, B^{J} a$ in $B^{l} a$ & $J \in\{2,3, \ldots \min (j, l)\}$ & $\min (j, l)-1$ \\
\hline & $\left\{a b^{J}, a B^{J}\right\}$ & $a b^{J}$ in $a b^{j}, a B^{J}$ in $a B^{l}$ & $J \in\{2,3, \ldots \min (j, l)\}$ & $\min (j, l)-1$ \\
\hline & $\left\{a b^{j} a, B^{j+1} a\right\}$ & $B^{j+1} a$ in $B^{l} a$ & $j<l$ & 1 \\
\hline & $\left\{a b^{j} a, a B^{j+1}\right\}$ & $a B^{j+1}$ in $a B^{l}$ & $j<l$ & 1 \\
\hline & $\left\{a b^{j} a, B^{j+2}\right\}$ & $B^{j+2}$ in $B^{l}$ & $j+2 \leq l$ & $l-j-1$ \\
\hline & $\left\{a B^{l} a, b^{l+1} a\right\}$ & $b^{l+1} a$ in $b^{j}$ & $j>l$ & 1 \\
\hline & $\left\{a B^{l} a, a b^{l+1}\right\}$ & $a b^{l+1}$ in $b^{j}$ & $j>l$ & 1 \\
\hline & $\left\{a B^{l} a, b^{l+2}\right\}$ & $b^{l+2}$ in $b^{j}$ & $j>l+1$ & $j-l-1$ \\
\hline & $\left\{a B^{l} a, a b^{j} a\right\}$ & & $j=l$ & 1 \\
\hline
\end{tabular}

Table 11: Linked pairs of $\left\langle a^{i} b^{j} a^{k} B^{l}\right\rangle$ of type III with $\mathrm{y}=b^{J}$.

\begin{tabular}{|c|c|c|c|c|}
\hline & configuration & with & if & add \\
\hline & $\left\{a^{I} b, B a^{I}\right\}$ & $a^{I}$ in $a^{i}$ & $I \in\{2,3, \ldots, i\}$ & $i-1$ \\
\hline & $\left\{b A^{I}, A^{I} B\right\}$ & $A^{I}$ in $A^{k}$ & $I \in\{2,3, \ldots, k\}$ & $k-1$ \\
\hline & $\left\{b^{I} A, a b^{I}\right\}$ & $b^{I}$ in $b^{j}$ & $I \in\{2,3, \ldots, j\}$ & $j-1$ \\
\hline & $\left\{A B^{I}, B^{I} a\right\}$ & $B^{I}$ in $B^{l}$ & $I \in\{2,3, \ldots, l\}$ & $l-1$ \\
\hline
\end{tabular}

Table 12: Linked pairs of $\left\langle a^{i} b^{j} A^{k} B^{l}\right\rangle$ of type II.

III. $\mathrm{y} \in\left\{a^{i}, b^{i}, A^{i}, B^{i}\right\}$ since the inverses of the subwords $\{a b, b A, A B, B a\}$ are $\{B A, a B, b a, A b\}$, which do not occur in $\left\langle a^{p} b^{q} A^{r} B^{s}\right\rangle$. It follows from Remark 3.3 and Table 2 that there are no linked pairs of this type.

Total of I, II, III:

$$
(i+k-2)(j+l-2)-i+j+k+l-4=(i+k-1)(j+l-1)-1
$$

\section{Proposition A.6.}

$$
\operatorname{SI}\left(\left\langle a^{i} b^{j} a^{k} b^{l} a^{m} B^{n}\right\rangle\right)=(i+k+m-1)(j+l+n-1)-2(k+\min (j, l)-1) .
$$

Proof. I. There are $(i+k+m-3)$ ocurrences of $a a,(j+l-2)$ ocurrences of $b b$ and $(n-1)$ occurrences of $B B$. This gives $(i+k+m-3)(j+l+n-3)$ linked pairs of type I.

II. y $\in\left\{a^{I} b^{J}, b^{J} a^{I}, a^{I} b^{J} a^{K}, b^{J}, B^{I}, a^{I}\right\}$ for some integers $I, J$ and $K$. By Remark 3.8 we do not need to consider words of the form rsusR.

(i) $\mathrm{y}=a^{I} b^{J}$. We analyze these linked pairs in Table 13, The total contribution of these pairs is 1 if $l>j$.

(ii) $\mathrm{y}=b^{J} a^{I}$. We analyze these pairs in Table 14. The total here is 1 if $l<j$. 


\begin{tabular}{|c|c|c|c|c|}
\hline & configuration & with & if & add \\
\hline & $\left\{a a^{I} b^{J} a, B a^{I} b^{J} b\right\}$ & & & not linked \\
\hline & $\left\{a a^{I} b^{j} a, b a^{k} b^{J} b\right\}$ & $a a^{I} b^{j} a$ in $a^{i} b^{j} a, b a^{k} b^{J} b$ in $b a^{k} b^{l}$ & $l>j$ and $i>k$ & 1 \\
\hline & $\left\{a a^{I} b^{J} b, B a^{i} b^{j} a\right\}$ & $a a^{I} b^{J} b$ in $a^{k} b^{l}$ & $l>j$ and $i<k$ & 1 \\
\hline & $\left\{B a^{i} b^{j} a, b a^{k} b^{J} b\right\}$ & $b a^{k} b^{J} b$ in $b a^{k} b^{l}$ & $l>j$ and $i=k$ & 1 \\
\hline & $\left\{b a^{I} b^{J} a, a a^{I} b^{J} b\right\}$ & & & not linked \\
\hline & $\left\{b a^{I} b^{J} a, B a^{i} b^{J} b\right\}$ & & & not linked \\
\hline
\end{tabular}

Table 13: Linked pairs of $\left\langle a^{i} b^{j} a^{k} b^{l} a^{m} B^{n}\right\rangle$ of type II with $\mathrm{y}=a^{I} b^{J}$.

\begin{tabular}{|c|c|c|c|c|}
\hline & configuration & with & if & add \\
\hline & $\left\{a b^{l} a^{I} a, b b^{J} a^{k} b\right\}$ & $a b^{l} a^{I} a$ in $a b^{I} a^{m}, b b^{J} a^{k} b$ in $b^{j} a^{k} b$ & $l<j$ and $k<m$ & 1 \\
\hline & $\left\{a b^{J} a^{I} a, b b^{J} a^{m} B\right\}$ & & & not linked \\
\hline & $\left\{a b^{J} a^{I} b, b b^{J} a^{I} a\right\}$ & & & not linked \\
\hline & $\left\{a b^{J} a^{I} b, b b^{J} a^{m} B\right\}$ & & & not linked \\
\hline & $\left\{a b^{l} a^{m} B, b b^{J} a^{k} b\right\}$ & $b b^{J} a^{k} b$ in $b^{j} a^{k} b$ & $l<j$ and $m=k$ & 1 \\
\hline \multirow{2}{*}{$a b^{l} a^{m} B, b b^{J} a^{I} a$} & $b b^{J} a^{I} a$ in $b^{j} a^{k}$ & $l<j$ and $m<k$ & 1 \\
\hline
\end{tabular}

Table 14: Linked pairs of $\left\langle a^{i} b^{j} a^{k} b^{l} a^{m} B^{n}\right\rangle$ of type II with $\mathrm{y}=b^{J} a^{I}$.

\begin{tabular}{|c|c|c|c|c|}
\hline & configuration & with & if & add \\
\hline & $\left\{B a^{i} b^{j} a^{I}, b a^{k} b^{l} a^{m} B\right\}$ & & & not linked (Remark [3.8) \\
\hline & $\left\{B a^{i} b^{j} a^{I}, a^{I} b^{l} a^{m} B\right\}$ & & & not linked \\
\hline & $\left\{B a^{i} b^{j} a^{k} b, a^{I} b^{l} a^{m} B\right\}$ & & & not linked (Remark [3.8) \\
\hline & $\left\{a^{I} b^{j} a^{I}, b a^{k} b^{l} a^{m} B\right\}$ & & & not linked (Remark 3.8) \\
\hline & $\left\{a^{I} b^{j} a^{k} b, b a^{k} b^{l} a^{m}\right\}$ & & & not linked \\
\hline
\end{tabular}

Table 15: Linked pairs of $\left\langle a^{i} b^{j} a^{k} b^{l} a^{m} B^{n}\right\rangle$ of type II with $\mathrm{y}=a^{I} b^{J} a^{K}$. 
(iii) $\mathrm{y}=a^{I} b^{J} a^{K}$. These pairs are analyzed in Table 15 where it is shown that there are no pairs of this type.

(iv) $\mathrm{y}=b^{I}$. These pairs are analyzed in Table [16. They contribute $|l-j|-1$ if $|l-j|>1$.

\begin{tabular}{|c|c|c|c|c|}
\hline & configuration & with & if & add \\
\hline & $\left\{b^{I}, a b^{j} a\right\}$ & $b^{I}$ in $b^{l}$ & $j+1<l$ & $l-j-1$ \\
\hline & $\left\{b^{I}, a b^{l} a\right\}$ & $b^{I}$ in $b^{j}$ & $l+1<j$ & $j-l-1$ \\
\hline & $\left\{a b^{I}, b^{I} a\right\}$ & & & not linked \\
\hline
\end{tabular}

Table 16: Linked pairs of $\left\langle a^{i} b^{j} a^{k} b^{l} a^{m} B^{n}\right\rangle$ of type II with $\mathrm{y}=b^{I}$,

(v) $\mathrm{y}=B^{I}$. The only possible pair, $\left\{a B^{I}, B^{I} a\right\}$ is not linked.

(vi) $\mathrm{y}=a^{I}$. Here (see Table2) the candidates for linked subwords are $\left\{a^{I} b, b a^{I}, B a^{I}, a^{I} B, a^{I}, b a^{I} b\right\}$. The number of occurrences of each possible pair is tabulated in Table 17. The total number is $2 m+2 i-4$.

\begin{tabular}{|c|c|c|c|c|}
\hline & configuration & with & if & add \\
\hline & $\left\{a^{I} b, B a^{I}\right\}$ & $B a^{I}$ in $B a^{i}, a^{I} b$ in $a^{i} b$ & $I \in\{2,3, \ldots, i\}$ & $i-1$ \\
\hline & $\left\{a^{I} b, B a^{I}\right\}$ & $B a^{I}$ in $B a^{i}, a^{I} b$ in $a^{k} b$ & $I \in\{2,3, \ldots, \min (i, k)\}$ & $\min (k, i)-1$ \\
\hline & $\left\{b a^{I}, a^{I} B\right\}$ & $a^{I} B$ in $a^{m} B, b a^{I}$ in $b a^{m}$ & $I \in\{2,3, \ldots, m\}$ & $m-1$ \\
\hline & $\left\{b a^{I}, a^{I} B\right\}$ & $a^{I} B$ in $a^{m} B, b a^{I}$ in $b a^{k}$ & $I \in\{2,3, \ldots, \min (k, m)\}$ & $\min (k, m)-1$ \\
\hline & $\left\{a a^{k} B, b a^{k} b\right\}$ & $a a^{k} B$ in $a^{m} B$ & $m>k$ & 1 \\
\hline & $\left\{B a^{k+1}, b a^{k} b\right\}$ & & $i>k$ & 1 \\
\hline & $\left\{b a^{k} b, a^{k+2}\right\}$ & $a^{k+2}$ in $a^{i}$ & $i>k+1$ & $i-k-1$ \\
\hline & $\left\{b a^{k} b, a^{k+2}\right\}$ & $a^{k+2}$ in $a^{m}$ & $m>k+1$ & $m-k-1$ \\
\hline
\end{tabular}

Table 17: Linked pairs of $\left\langle a^{i} b^{j} a^{k} b^{l} a^{m} B^{n}\right\rangle$ of type II with $\mathrm{y}=a^{I}$.

The total number of linked pairs of type II is $|j-l|+2 m+2 i-4$.

III. y $=b^{I}$. Analysis: Table [18, using Remark 3.3 and Table 2, The values in Table 18 may be grouped as follows: $(\mathrm{a}+\mathrm{b}+\mathrm{e}+\mathrm{f}+\mathrm{i}+\mathrm{j})=2 n-2,(\mathrm{~d}+\mathrm{k}+\mathrm{q})=j-1$, $(\mathrm{c}+\mathrm{l}+\mathrm{r})=l-1,(\mathrm{~g}+\mathrm{h}+\mathrm{m}+\mathrm{n}+\mathrm{o}+\mathrm{p})=2$.

Total for types I, II, III:

$$
(i+k+m-3)(j+l+n-3)+|j-l|+2 m+2 i-4+2 n+j+l-2 .
$$

Applying $|j-l|=\max (j, l)-\min (j, l)$ and $j+l=\max (j, l)+\min (j, l)$ yields the desired result. 


\begin{tabular}{|c|c|c|c|c|}
\hline & configuration & with & if & $\overline{\text { add }}$ \\
\hline $\mathrm{a}$ & $\left\{B^{K} a, b^{K} a\right\}$ & $B^{K}$ in $B^{n}, b^{K}$ in $b^{l}$ & $K \in\{2,3, \ldots, \min (n, l)\}$ & $\min (n, l)-1$ \\
\hline $\mathrm{b}$ & $\left\{B^{K} a, b^{K} a\right\}$ & $B^{K}$ in $B^{n}, b^{K}$ in $b^{j}$ & $K \in\{2,3, \ldots, \min (n, j)\}$ & $\min (n, j)-1$ \\
\hline $\mathrm{c}$ & $\left\{a B^{K}, a b^{K}\right\}$ & $B^{K}$ in $B^{n}, b^{K}$ in $b^{l}$ & $K \in\{2,3, \ldots, \min (n, l)\}$ & $\min (n, l)-1$ \\
\hline $\mathrm{d}$ & $\left\{a B^{K}, a b^{K}\right\}$ & $B^{K}$ in $B^{n}, b^{K}$ in $b^{j}$ & $K \in\{2,3, \ldots, \min (n, j)\}$ & $\min (n, j)-1$ \\
\hline $\mathrm{e}$ & $\left\{B^{K} a, a b^{j} a\right\}$ & $B^{K}$ in $B^{n}$ & $n>j$ & 1 \\
\hline $\mathrm{f}$ & $\left\{B^{K} a, a b^{l} a\right\}$ & $B^{K}$ in $B^{n}$ & $n>l$ & 1 \\
\hline $\mathrm{g}$ & $\left\{a B^{K}, a b^{j} a\right\}$ & $B^{K}$ in $B^{n}$ & $n>j$ & 1 \\
\hline $\mathrm{h}$ & $\left\{a B^{K}, a b^{l} a\right\}$ & $B^{K}$ in $B^{n}$ & $n>l$ & 1 \\
\hline $\mathrm{i}$ & $\left\{B^{K}, a b^{j} a\right\}$ & $B^{K}$ in $B^{n}$ & $n>j+1$ & $n-j-1$ \\
\hline $\mathrm{j}$ & $\left\{B^{K}, a b^{l} a\right\}$ & $B^{K}$ in $B^{n}$ & $n>l+1$ & $n-l-1$ \\
\hline $\mathrm{k}$ & $\left\{a B^{n} a, b b^{K} a\right\}$ & $b b^{K}$ in $b^{j}$ & $j>n$ & 1 \\
\hline l & $\left\{a B^{n} a, b b^{K} a\right\}$ & $b b^{K}$ in $b^{l}$ & $l>n$ & 1 \\
\hline $\mathrm{m}$ & $\left\{a B^{n} a, a b^{K} b\right\}$ & $b^{K} b$ in $b^{j}$ & $j>n$ & 1 \\
\hline $\mathrm{n}$ & $\left\{a B^{n} a, a b^{K} b\right\}$ & $b^{K} b$ in $b^{l}$ & $l>n$ & 1 \\
\hline $\mathrm{O}$ & $\left\{a B^{n} a, a b^{j} a\right\}$ & & $n=j$ & 1 \\
\hline $\mathrm{p}$ & $\left\{a B^{n} a, a b^{l} a\right\}$ & & $n=l$ & 1 \\
\hline $\mathrm{q}$ & $\left\{a B^{n} a, b^{K}\right\}$ & $b^{K}$ in $b^{j}$ & $j>n+1$ & $j-n-1$ \\
\hline $\mathrm{r}$ & $\left\{a B^{n} a, b^{K}\right\}$ & $b^{K}$ in $b^{l}$ & $l>n+1$ & $l-n-1$ \\
\hline
\end{tabular}

Table 18: Linked pairs of $\left\langle a^{i} b^{j} a^{k} b^{l} a^{m} B^{n}\right\rangle$ of type III with $\mathrm{y}=b^{K}$.

Proposition A.7. IN $\left(\left\langle a^{i} b^{j} a^{k} b^{l}\right\rangle,\left\langle a^{m} B^{n}\right\rangle\right)=(i+k) n+m(j+l)$

Proof. I. There are $(i+k-2)(n-1)+(m-1)(j+l-2)$ of these.

II. $\mathrm{y}=a^{K}$ for some positive integer $K$. Analysis: Table 19, using Table 22, The values in Table 19 may be grouped as follows: $(\mathrm{a}+\mathrm{e}+\mathrm{g})=i-1,(\mathrm{~b}+\mathrm{f}+\mathrm{h})=k-1$, $(\mathrm{c}+\mathrm{d}+\mathrm{k}+\mathrm{l}+\mathrm{o}+\mathrm{p})=2 m-2$, and $(\mathrm{i}+\mathrm{j}+\mathrm{m}+\mathrm{n}+\mathrm{p}+\mathrm{q})=2$

III. $\mathrm{y}=b^{K}$. Analysis: Table 20, using Table 2 (interchanging the roles of $a$ 's and $b$ 's) and Remark 3.3. The values group in the following way: $(\mathrm{a}+\mathrm{b}+\mathrm{e}+\mathrm{f}+\mathrm{k}+\mathrm{l})=$ $2 n-2,(\mathrm{c}+\mathrm{m}+\mathrm{q})=j-1,(\mathrm{~d}+\mathrm{p}+\mathrm{r})=l-1,(\mathrm{~g}+\mathrm{h}+\mathrm{i}+\mathrm{j}+\mathrm{n}+\mathrm{o})=2$.

Grand Total: $(i+k-2)(n-1)+(m-1)(j+l-2)+i+j+k+l+2 m+2 n-4=$ $(i+k) n+m(j+l)$.

\section{References}

[1] A. Basmajian, The stable neighborhood theorem and lengths of closed geodesics, Proc. Amer. Math. Soc. 119(1) (1993) 217-224. 


\begin{tabular}{|c|c|c|c|c|}
\hline & configuration & with & if & add \\
\hline $\mathrm{a}$ & $\left\{a^{K} b, B a^{K}\right\}$ & $a^{K} b$ in $a^{i} b$ & $K \in\{2,3, \ldots, \min (m, i)\}$ & $\min (m, i)-1$ \\
\hline $\mathrm{b}$ & $\left\{a^{K} b, B a^{K}\right\}$ & $a^{K} b$ in $a^{k} b$ & $K \in\{2,3, \ldots, \min (m, k)\}$ & $\min (m, k)-1$ \\
\hline $\mathrm{c}$ & $\left\{a^{i} B, b a^{i}\right\}$ & $b a^{K}$ in $b a^{i}$ & $K \in\{2,3, \ldots, \min (m, i)\}$ & $\min (m, i)-1$ \\
\hline $\mathrm{d}$ & $\left\{a^{i} B, b a^{i}\right\}$ & $b a^{K}$ in $b a^{k}$ & $K \in\{2,3, \ldots, \min (m, k)\}$ & $\min (m, k)-1$ \\
\hline $\mathrm{e}$ & $\left\{a^{m+2}, B a^{m} B\right\}$ & $a^{m+2}$ in $a^{i}$ & $m+2 \leq i$ & $i-m-1$ \\
\hline $\mathrm{f}$ & $\left\{a^{m+2}, B a^{m} B\right\}$ & $a^{m+2}$ in $a^{k}$ & $m+2 \leq k$ & $k-m-1$ \\
\hline $\mathrm{g}$ & $\left\{a^{m+1} b, B a^{m} B\right\}$ & $a^{m+1} b$ in $a^{i} b$ & $m<i$ & 1 \\
\hline $\mathrm{h}$ & $\left\{a^{m+1} b, B a^{m} B\right\}$ & $a^{m+1} b$ in $a^{j} b$ & $m<k$ & 1 \\
\hline $\mathrm{i}$ & $\left\{b a^{m+1}, B a^{m} B\right\}$ & $b a^{m+1}$ in $b a^{i}$ & $m<i$ & 1 \\
\hline $\mathrm{j}$ & $\left\{b a^{m+1}, B a^{m} B\right\}$ & $b a^{m+1}$ in $b a^{j}$ & $m<k$ & 1 \\
\hline $\mathrm{k}$ & $\left\{a^{k+1} B, b a^{k} b\right\}$ & $a^{k+1} B$ in $a^{m}$ & $k<m$ & 1 \\
\hline $\mathrm{l}$ & $\left\{a^{i+1} B, b a^{i} b\right\}$ & $a^{i+1} B$ in $a^{m} B$ & $k<m$ & 1 \\
\hline $\mathrm{m}$ & $\left\{B a^{k+1}, b a^{k} b\right\}$ & $B^{k+1} a$ in $B a^{m}$ & $k<m$ & 1 \\
\hline $\mathrm{n}$ & $\left\{B a^{i+1}, b a^{i} b\right\}$ & $B^{i+1} a$ in $B a^{m}$ & $k+2 \leq m$ & $m-i-1$ \\
\hline $\mathrm{o}$ & $\left\{a^{i+2}, b a^{i} b\right\}$ & $a^{i+2}$ in $a^{m}$ & $k+2 \leq m$ & $m-k-1$ \\
\hline $\mathrm{p}$ & $\left\{a^{i+2}, b a^{k} b\right\}$ & $a^{i+2}$ in $a^{m}$ & $k=m$ & 1 \\
\hline $\mathrm{q}$ & $\left\{b a^{i} b, B a^{m} B\right\}$ & & & \\
\hline $\mathrm{r}$ & $\left\{b a^{k} b, B a^{m} B\right\}$ & & $k+m$ & 1 \\
\hline
\end{tabular}

Table 19: Linked pairs of $\left\langle a^{i} b^{j} a^{k} b^{l}\right\rangle$ and $\left\langle a^{m} B^{n}\right\rangle$ of type II with $\mathrm{y}=a^{K}$.

\begin{tabular}{|c|c|c|c|c|}
\hline & configuration & with & if & add \\
\hline $\mathrm{a}$ & $\left\{a b^{K}, a B^{K}\right\}$ & $b^{K}$ in $b^{j}$ & $K \in\{2,3, \ldots, \min (j, n)\}$ & $\min (j, n)-1$ \\
\hline $\mathrm{b}$ & $\left\{a b^{K}, a B^{K}\right\}$ & $b^{K}$ in $b^{l}$ & $K \in\{2,3, \ldots, \min (l, n)\}$ & $\min (l, n)-1$ \\
\hline $\mathrm{c}$ & $\left\{b^{K} a, B^{K} a\right\}$ & $b^{K} a$ in $b^{j} a$ & $K \in\{2,3, \ldots, \min (j, n)\}$ & $\min (j, n)-1$ \\
\hline $\mathrm{d}$ & $\left\{b^{K} a, B^{K} a\right\}$ & $b^{K} a$ in $b^{l} a$ & $K \in\{2,3, \ldots, \min (l, n)\}$ & $\min (l, n)-1$ \\
\hline $\mathrm{e}$ & $\left\{a b^{l} a, B^{l+1} a\right\}$ & & $l<n$ & 1 \\
\hline $\mathrm{f}$ & $\left\{a b^{j} a, B^{j+1} a\right\}$ & & $j<n$ & 1 \\
\hline $\mathrm{g}$ & $\left\{a b^{j} a, a B^{j+1}\right\}$ & & $j<n$ & 1 \\
\hline $\mathrm{h}$ & $\left\{a b^{l} a, a B^{l+1}\right\}$ & & $l<n$ & 1 \\
\hline $\mathrm{i}$ & $\left\{a b^{j} a, a B^{n} a\right\}$ & & $j=n$ & 1 \\
\hline $\mathrm{j}$ & $\left\{a b^{l} a, a B^{n} a\right\}$ & & $j+2 \leq n$ & $n-j-1$ \\
\hline $\mathrm{k}$ & $\left\{a b^{j} a, B^{j+2}\right\}$ & & $l+2 \leq n$ & $n-l-1$ \\
\hline $\mathrm{l}$ & $\left\{a b^{l} a, B^{l+2}\right\}$ & & $n<j$ & 1 \\
\hline $\mathrm{m}$ & $\left\{b^{n} a, a B^{n} a\right\}$ & $b^{n} a$ in $b^{j} a$ & $n<l$ & 1 \\
\hline $\mathrm{n}$ & $\left\{b^{n} a, a B^{n} a\right\}$ & $b^{n} a$ in $b^{l} a$ & $n<j$ & 1 \\
\hline $\mathrm{o}$ & $\left\{a b^{K}, a B^{K} a\right\}$ & & $n<l$ & 1 \\
\hline $\mathrm{p}$ & $\left\{a b^{K}, a B^{K} a\right\}$ & & $n+2 \leq j$ & $l-n-1$ \\
\hline $\mathrm{q}$ & $\left\{b^{n+2}, a B^{n} a\right\}$ & $b^{n+2} a$ in $b^{j}$ & $n+2 \leq l$ & \\
\hline $\mathrm{r}$ & $\left\{b^{n+2}, a B^{n} a\right\}$ & $b^{n+2} a$ in $b^{l}$ & &
\end{tabular}

Table 20: Linked pairs of $\left\langle a^{i} b^{j} a^{k} b^{l}\right\rangle$ and $\left\langle a^{m} B^{n}\right\rangle$ of type III with $\mathrm{y}=b^{K}$. 
[2] J. Birman and C. Series, An algorithm for simple curves on surfaces, J. London Math. Soc. (2), 29 (1984), 331-342.

[3] A. Blood, The maximal number of transverse self-intersections on the punctured torus, Proceedings of the REU Program in Mathematics, Corvallis OR, August 2002. Link to report.

[4] M. Bridson and A. Haefliger, Metric Spaces of Non-Positive Curvature, SpringerVerlag, Berlin Heidelberg New York, 1991.

[5] P. Buser, Geometry and Spectra of Compact Riemann Surfaces, Birkhauser Boston, 1992.

[6] M. Chas, Combinatorial Lie bialgebras of curves on surfaces, Topology 43 (2004) 543-568. arXiv: 0105178v2 [math.GT]

[7] F. Chemotti and A. Rau, Intersection numbers of closed curves on the punctured torus, Proceedings of the REU Program in Mathematics, Corvallis OR, August 2004. Link to report.

[8] http://www.math.sunysb.edu/ moira/CLB/CLB09/

[9] M. Cohen and M. Lustig, Paths of geodesics and geometric intersection numbers I, Combinatorial Group Theory and Topology, Alta, Utah, 1984, Ann. of Math. Studies 111, Princeton Univ. Press, Princeton, (1987), 479-500.

[10] J. Hass and P. Scott, Intersections of curves on surfaces, Israel J. Math. 51 (1985) 90-120.

[11] S. Lalley, Renewal theorems in symbolic dynamics, with applications to geodesic flows, noneuclidean tessellations, and their fractal limits.Acta. Math. 163 (1989), $1-55$.

[12] S. Lalley, Self-intersections of closed geodesics on a negatively curved surface: statistical regularities, Convergence in ergodic theory and probability (Columbus, OH, 1993), Ohio State Univ. Math. Res. Inst. Publ., 5, de Gruyter, Berlin, 1996, pp. 263-272. MR1412610 (97h:58103)

[13] R. Lyndon and R. Schupp, Combinatorial Group Theory, Springer-Verlag, Berlin Heidelberg New York, 2001.

[14] G. Margulis, Applications of ergodic theory to the investigation of manifolds of negative curvature, Funct. Anal. and Appl. 3 (1983) 573-591.

[15] G. McShane and I. Rivin, A norm on homology of surfaces and counting simple geodesics, Internat. Math. Res. Notices, February 1995. MR 96b:57014 
[16] J. Milnor, A note on curvature and the fundamental group, J. Differential Geometry 2 (1968) 1-7.

[17] M. Mirzakhani, Growth of the number of simple closed geodesics on a hyperbolic surface, Ann. of Math., to appear.

[18] W. Parry and M. Pollicott, An analogue of the prime-number theorem for closed orbits of Axiom A flows, Ann. of Math., 118 (1983), 573-591.

[19] I. Rivin, Simple curves on surfaces, Geom. Dedicata 87 (2001), 345-360.

[20] I. Rivin, Geodesics with one self-intersection and other stories, arXiv: 0901.2543 [math.GT]

[21] S. Tan, Self-intersections of curves on surfaces, Geom. Dedicata 62 no. 2 (1996) 209-225.

[22] D. Thurston, On geometric intersection of curves in surfaces, preprint, http://www.math.columbia.edu/ dpt/writing.html

Department of Mathematics, Stony Brook University, Stony Brook, NY, 11794.

E-mail address: moira@math.sunysb.edu, tony@math.sunysb.edu 